\title{
Cell-Centric Heuristics for the Classification of Cellular Automata
}

\author{
Valmir C. Barbosa* \\ Fernando M. N. Miranda \\ Matheus C. M. Agostini \\ Universidade Federal do Rio de Janeiro \\ Programa de Engenharia de Sistemas e Computação, COPPE \\ Caixa Postal 68511 \\ 21941-972 Rio de Janeiro - RJ, Brazil
}

November 20, 2018

\begin{abstract}
We study the classification of cellular-automaton update rules into Wolfram's four classes. We start with the notion of the input entropy of a spatiotemporal block in the evolution of a cellular automaton, and build on it by introducing two novel entropy measures, one that is also based on inputs to the cells, the other based on state transitions by the cells. Our two new entropies are both targeted at the classification of update rules by parallel machines, being therefore mindful of the necessary communications requirements; we call them cell-centric input entropy and cell-centric transition entropy to reflect this fact. We report on extensive computational experiments on both one- and two-dimensional cellular automata. These experiments allow us to conclude that the two new entropies possess strong discriminatory capabilities, therefore providing valuable aid in the classification process.
\end{abstract}

Keywords: Classification of cellular automata, Input entropy, Parallel simulation of cellular automata.

\section{Introduction}

Cellular automata are discrete-time dynamical systems comprising finite-state units, called cells, whose states evolve in time as a result of the interactions with other cells. Since their introduction nearly five decades ago by von Neumann [1,

${ }^{*}$ Corresponding author (valmir@cos.ufrj.br). 
cellular automata have acquired an ever more prominent status as a modeling tool in several research areas (cf., e.g., [2, 3] and the references therein), and have even come to be regarded by some as a central abstraction in the modeling of nature's fundamental processes [4].

For $S=\{0, \ldots, s-1\}$ the set of possible states, and for $t \geq 0$ an integer, a cellular automaton with $n$ cells evolves from time $t$ to time $t+1$ by synchronously updating all $n$ states by the application of a deterministic mapping $F_{f}$ from $S^{n}$ to $S^{n}$. This mapping $F_{f}$ is global in nature and depends on the local update rule $f$, which dictates how each individual state is to be updated given the cell's state at time $t$ as well as the states of those cells that lie within a neighborhood of size $\delta$. The update rule $f$ is then a mapping from $S^{1+\delta}$ to $S$.

Normally a cell's neighborhood in a cellular automaton is determined by an underlying multidimensional lattice according to several possible criteria. For example, a cell's neighbors relative to a certain dimension of the lattice may be taken to be those cells that are $r>0$ edges away along that dimension but no edges away along any other dimension, $r$ being usually referred to as the radius of the update rule in that dimension. For unit radii in all dimensions, this characterizes what is known as the von Neumann neighborhood, but in this paper we employ the same denomination also for greater radii. Another example neighborhood comes from letting two cells be neighbors of each other whenever one can be reached from the other by treading no more edges along a certain dimension than the update rule's radius along that dimension. For unit radii this is the Moore neighborhood, but once again we generalize and in this paper employ the same denomination under greater radii as well. When $n$ is finite, it is customary to regard the lattice as having cylindrical boundaries, that is, as allowing every cell to have exactly two nearest neighbors along each dimension.

Finite cellular automata, those for which $n$ is finite, are necessarily such that $F_{f}$ eventually leads to a fixed point, or a limit cycle, of configurations in $S^{n}$, that is, either $x$ such that $F_{f}(x)=x$ or $x_{0}, \ldots, x_{p-1}$, with $p>0$, such that $x_{0}=F_{f}\left(x_{p-1}\right), x_{1}=F_{f}\left(x_{0}\right)$, and so on [5]. The case of infinite cellular automata, on the other hand, is far more complicated and intriguing, since now $n$ is formally infinite and no periodicity is guaranteed to emerge from the successive application of $F_{f}$.

Both in the finite and in the infinite cases, cellular automata have along the years been the subject of theoretical and experimental analyses. For a summary of key results, the reader is referred, for example, to [6] 7] and to their many references. One of the most appealing topics of investigation has been the classification of the update rule $f$, and consequently of the cellular automata based on it, regarding its "complexity."

Interest in this question received its initial impetus from the study by Wolfram of infinite one-dimensional cellular automata [8], which resulted in the empirical finding that, nearly regardless of initial states, $f$ consistently falls within one of four possible qualitative categories: (i) evolution leads to a homogeneous configuration, i.e., a configuration in which all cells have the same state; (ii) evolution leads to an inhomogeneous fixed point or to a limit cycle; (iii) evo- 
lution leads to a chaotic succession of configurations; or (iv) evolution leads to complex localized spatiotemporal structures that are "sometimes long-lived." Although initially conceived for the one-dimensional case, there is in principle no reason why such a qualitative classification should not also be applicable to higher-dimensional cases. In fact, similar studies for the two-dimensional case have appeared as early as in 9 .

Naturally, class-(iv) update rules are intuitively associated with the realization of "complex" computations by the cellular automata that are built on them, that is, precisely those computations that underlie so much of the interest in cellular automata as modeling tools. Not surprisingly, then, considerable effort has been channeled into finding approaches to automatically categorize update rules into the classes (i)-(iv). Formally, all such efforts hover around the so-called limit set of an update rule $f$ in the infinite case, which is the set of configurations that result from all possible initial configurations after the passage of arbitrarily long time. As it turns out, every nontrivial property of a limit set (i.e., a property that holds for at least one cellular automaton and does not hold for at least one other) can be proven undecidable through a reduction from the problem of whether a limit set is a singleton [10, itself known to be undecidable [1].

As a consequence of this inherent undecidability, every effective strategy for categorizing update rules must necessarily be of a heuristic nature or else eventually boil down to a heuristic if it is to have any practical use. Our interest in this paper is the study of heuristics that can be coupled with the parallel simulation of cellular automata in order to analyze the spatiotemporal patterns that emerge, aiming at categorizing the underlying update rule within Wolfram's

four classes. Efficiency in the form of minimal communications needs is then an essential requirement, leading to what we term cell-centric heuristics, that is, heuristics that depend as minimally as possible on the exchange of information among processors during the simulation of a cellular automaton.

We start in Section 2 with a review of some of the prominent heuristics that have been proposed for automatically classifying update rules, and proceed in Section 3 to a discussion of the so-called input-entropy measures. Our cellcentric heuristics are presented in Section 4 with results from computational experiments on one- and two-dimensional cellular automata given in Section 5 . Further considerations on the computational results appear in Section [6] and concluding remarks come in Section 7

\section{Background}

In broad terms, we distinguish two essential kernel classes of strategies for the categorization of update rules. The first class comprises those techniques that aim at extracting the update rules' computational capabilities by solely considering the update rule itself, not simulations of cellular automata for examination of the resulting spatiotemporal patterns. Approaches of this type have concen- 
trated on one-dimensional cellular automata, so a cell's neighborhood size is in fact $\delta=2 r$.

The pioneering step within this class of approaches was taken by Langton [12, who proposed to classify an update rule $f$ into the four Wolfram classes by examining a single parameter, denoted by $\lambda_{f}$ and given by

$$
\lambda_{f}=1-\frac{q}{s^{1+2 r}} .
$$

In (11), $q$ is the number of distinct $(1+2 r)$-tuples on which $f$ outputs $\sigma$, where $\sigma \in S$ is any of the so-called quiescent states, that is, $\sigma$ is such that $f(\sigma, \ldots, \sigma)=$ $\sigma$.

The initial report on the use of the $\lambda_{f}$ parameter indicated that it behaves as an order parameter with respect to which a phase transition occurs: on generating update rules $f$ with increasing $\lambda_{f}$ one first encounters class-(i) behavior, then class (ii), then class (iv) around $\lambda_{f}=0.5$, then finally class-(iii) behavior. This seemed to suggest that complexity was to be found at the region in the $\lambda_{f}$ space that became known as the "edge of chaos." But, in addition to the obvious difficulties regarding the existence and choice of a quiescent state, criticism regarding the existence and nature of the purported phase transition soon came from several sources (cf., e.g., 13, 14, 15]). In particular, it now appears that update rules belonging to several classes, not just class (iv), are to be found near $\lambda_{f}=0.5$.

Two other interesting approaches have been introduced that are also of the same nature in that they also dispense with the need for computer simulations of cellular automata. The two approaches share the goal of investigating how the information contained in an update rule $f$ affects the overall behavior of cellular automata built on $f$. One of the approaches is topological in nature, that is, it seeks to analyze a cellular automaton's global behavior by identifying finite-size spatial patterns and characterizing their appearance and evolution [16. By contrast, the other one [17 is algorithmic and aims at characterizing update rule $f$ from the perspective of Kolmogorov complexity [18, that is, the perspective of the shortest possible description of $f$. Both approaches relate clearly to the Wolfram classification, while at the same time shedding new light on it, each from its particular perspective. The latter approach, in addition, may also hold a key to some of the incongruities that are inherent to Langton's parameter $\lambda_{f}$. It is worth remarking, however, that because each of the two approaches induces its own class system, neither one is found to relate clearly to class (iv). The reader is referred to the original sources for details.

A wholly distinct class of strategies to categorize cellular-automaton update rules concentrates on the examination of space-time patterns as they appear during the evolution of cellular automata from as representative a sample of initial configurations as possible. Now, of course, the fact that the cellular automata under examination are formally infinite has to be reckoned with; we will come to this later, and will for now ignore any difficulties that such infinities may cause in practice. We do mention, however, that some successful approaches are built from the start on the assumption that $n$ is finite. One example is the "com- 
putational mechanics" exemplified in [19], which for one-dimensional cellular automata draws heavily on finite-state machines [20] derived from patterns in the cellular automaton's evolution to characterize the fundamental spatiotemporal features that are inherent to each update rule.

The approaches in this class that are central to our interest are those that rely on some form of entropy measure as the basis of the categorization effort. The initial approach along these lines appeared in the same paper that introduced the four-class Wolfram classification [8]. Essentially, what it does is to consider the probability distribution of space-time blocks as they occur during the evolution of cellular automata for a fixed update rule $f$ and then use this distribution to define the desired entropies.

For a more precise characterization, let $d>0$ be the number of dimensions of the cellular automata in question, and let $X_{1}, \ldots, X_{d}$ denote numbers of contiguous cells along each dimension. For $T>0$ a number of successive time steps during an evolution of the cellular automaton that employs update rule $f$, we need the probability, given $f$, that an $X_{1} \times \cdots \times X_{d} \times T$ block of states appears somewhere in the spatiotemporal trace of the cellular automaton's evolution. Clearly, the number of possible blocks is $s^{X_{1} \ldots X_{d} T}$. We denote by $P_{i}$ the probability of the $i$ th block, $1 \leq i \leq s^{X_{1} \ldots X_{d} T}$.

Two basic entropies can now be defined. These are the set entropy

$$
E_{f}\left(X_{1}, \ldots, X_{d}, T\right)=\frac{1}{T} \log _{s}\left(\sum_{i=1}^{s^{X_{1} \ldots X_{d} T}} \theta\left(P_{i}\right)\right),
$$

where $\theta(p)=1$ for $p>0$ and $\theta(0)=0$, and the measure entropy

$$
E_{f}^{\mu}\left(X_{1}, \ldots, X_{d}, T\right)=-\frac{1}{T} \sum_{i=1}^{s^{X_{1} \ldots X_{d} T}} P_{i} \log _{s} P_{i} .
$$

From them, we obtain the limiting quantities

$$
H_{f}=\lim _{\substack{X_{1}, \ldots, X_{d} \rightarrow \infty \\ T / X_{1}, \ldots, T / X_{d} \rightarrow \infty}} E_{f}\left(X_{1}, \ldots, X_{d}, T\right)
$$

and

$$
H_{f}^{\mu}=\lim _{\substack{X_{1}, \ldots, X_{d} \rightarrow \infty \\ T / X_{1}, \ldots, T / X_{d} \rightarrow \infty}} E_{f}^{\mu}\left(X_{1}, \ldots, X_{d}, T\right)
$$

respectively.

The quantity in (44) gives the asymptotic rate at which the diversity of spatiotemporal patterns increases with time, and the one in (5) represents the average amount of "new information" that each new configuration of the cellular automaton contributes as time elapses. As it turns out, these quantities (or variations thereof obtained by taking the limit exclusively as $X_{1}, \ldots, X_{d} \rightarrow \infty$ while $T$ is kept constant, or as $T \rightarrow \infty$ while $X_{1}, \ldots, X_{d}$ are kept constant) yield 
insight into how to categorize $f$. Coarsely, all indicators vanish for class-(i) cellular automata and are nonzero for class-(iii) cellular automata. Discriminating class (ii), in turn, requires decoupling space and time: indicators resulting from letting $T \rightarrow \infty$ alone are zero, while those related to letting $X_{1}, \ldots, X_{d} \rightarrow \infty$ for fixed $T$ are nonzero. As for class (iv), once again the attempt at identification is eluded.

\section{Input entropy}

The concept of input entropy is due to Wuensche 21] and constitutes an attempt to merge together some of the key features of the two classes of strategies discussed in Section 2 those that seek to base update-rule classification on examining the update rule solely and those that rely on space-time signatures of evolving cellular automata. Given one of the $X_{1} \times \cdots \times X_{d} \times T$ state blocks of that section, we start by considering the probability that, inside the block, each of the possible $s^{1+\delta}$ inputs to a cell occurs. Denoting the probability of the $i$ th input by $Q_{i}, 1 \leq i \leq s^{1+\delta}$, the input entropy is defined by

$$
I_{f}\left(X_{1}, \ldots, X_{d}, T\right)=-\sum_{i=1}^{s^{1+\delta}} Q_{i} \log _{s} Q_{i} .
$$

The use of the input entropy in practice starts by fixing the values of $X_{1}, \ldots, X_{d}$ and of $T$ and then choosing the $X_{1} \ldots X_{d}$ cells to be observed during simulations of the cellular automaton built on $f$. Each simulation is conducted from a randomly selected initial configuration and runs for $t_{+}$time steps, for some $t_{+} \geq T-1$, generating a new configuration at each time step. For each time $t$ in the interval $\left[t_{0}, t_{+}\right]$with $T-1 \leq t_{0} \leq t_{+}$, the probability $Q_{i}$ that the $i$ th input occurs within a $X_{1} \times \cdots \times X_{d} \times T$ block can be approximated by $q_{i}^{t} / X_{1} \ldots X_{d} T$, where $q_{i}^{t}$ is the number of occurrences of the $i$ th input within the block that ends at time $t$. The practical entropy figure that stems from (6) is then, for the block that ends at time $t$,

$$
I_{f}^{t}\left(X_{1}, \ldots, X_{d}, T\right)=-\sum_{i=1}^{s^{1+\delta}}\left(\frac{q_{i}^{t}}{X_{1} \ldots X_{d} T}\right) \log _{s}\left(\frac{q_{i}^{t}}{X_{1} \ldots X_{d} T}\right) .
$$

The mean and variance of the quantity in (17) for $t=t_{0}, \ldots, t_{+}$, that is,

$$
\overline{I_{f}}\left(X_{1}, \ldots, X_{d}, T\right)=\frac{1}{t_{+}-t_{0}+1} \sum_{t=t_{0}}^{t_{+}} I_{f}^{t}\left(X_{1}, \ldots, X_{d}, T\right)
$$

and

$$
\begin{aligned}
& \sigma^{2}\left(I_{f}\left(X_{1}, \ldots, X_{d}, T\right)\right) \\
& \quad=\frac{1}{t_{+}-t_{0}+1} \sum_{t=t_{0}}^{t_{+}}\left[I_{f}^{t}\left(X_{1}, \ldots, X_{d}, T\right)-\overline{I_{f}}\left(X_{1}, \ldots, X_{d}, T\right)\right]^{2},
\end{aligned}
$$


respectively, can be used to reveal the Wolfram class to which update rule $f$ belongs, after having themselves been averaged over some number of simulations for randomly chosen initial configurations. Roughly, for $d=1$ it has been found that low means and variances bespeak class-(i) or (ii) behavior, while high means and low variances indicate a class-(iii) update rule in action. Class-(iv) behavior is characterized by medium-valued means and high variances [21].

\section{Cell-centric heuristics}

Computing the mean and variance of the input entropy as indicated respectively in (8) and (9) requires simulating the cellular automaton that is based on $f$ for $t_{+}$ time steps and accumulating the quantity given in (17) while an $X_{1} \times \cdots \times X_{d} \times T$ block "window" is slid from an initial position that makes the block end at time $t_{0}$ through a final position at which the block ends at time $t_{+}$. When the simulation is performed in parallel, the $X_{1} \ldots X_{d}$ cells do not all reside at the same processor, so computing the input entropy as given by (7) for all values of $t$ requires a considerable amount of communication involving the processors that lodge the cells.

Given that one processor, call it $P$, has been singled out for coalescing all the information required for computing the mean and the variance, in essence the number of integers that needs to be communicated to $P$ is $O\left(X t_{+} \delta\right)$, where $X$ is the number of cells allocated outside $P$. In the case of true massive parallelism (one cell per processor), this becomes $O\left(X_{1} \ldots X_{d} t_{+} \delta\right)$. In general, for each cell and each time $t$, the integers to be communicated are the $1+\delta$ integers needed for specifying the input to that cell at time $t$. If that input is the $i$ th possible input, then communicating the $1+\delta$ integers contributes one unit to each of

$q_{i}^{t}, \ldots, q_{i}^{t+T-1}$; that is, it contributes to the calculation of the input entropy of (7) for $T$ blocks (the one ending at time $t$ through the one ending at time $t+T-1)$.

\subsection{Cell-centric input entropy}

The crux of the $O\left(X t_{+} \delta\right)$ communications requirement is that the logarithm appearing in (7) can only be assessed after the contributions to $q_{i}^{t}$ have been taken into account for all $X_{1} \ldots X_{d}$ cells, in particular for all the $X$ cells lodged outside processor $P$. The first step towards obtaining a cell-centric approximation to (7), one that allows communications requirements to be reduced dramatically, is to examine more closely the argument to that logarithm and to notice that $q_{i}^{t} / X_{1} \ldots X_{d}$ is the average number of occurrences of the $i$ th input per cell within the $X_{1} \times \cdots \times X_{d} \times T$ block.

Let $q_{i}^{c, t}$ denote the number of occurrences of the $i$ th input for the $c$ th cell inside the block that ends at time $t$; clearly, $q_{i}^{t}=\sum_{c=1}^{X_{1} \ldots X_{d}} q_{i}^{c, t}$ and (7) can be rewritten as 


$$
\begin{aligned}
I_{f}^{t}\left(X_{1}, \ldots, X_{d}, T\right) \\
=-\sum_{i=1}^{s^{1+\delta}} \frac{\sum_{c=1}^{X_{1} \ldots X_{d}} q_{i}^{c, t}}{X_{1} \ldots X_{d} T} \log _{s}\left(\frac{\sum_{c=1}^{X_{1} \ldots X_{d}} q_{i}^{c, t}}{X_{1} \ldots X_{d} T}\right) \\
=-\frac{1}{X_{1} \ldots X_{d}} \sum_{c=1}^{X_{1} \ldots X_{d}} \sum_{i=1}^{s^{1+\delta}}\left(\frac{q_{i}^{c, t}}{T}\right) \log _{s}\left(\frac{\sum_{c^{\prime}=1}^{X_{1} \ldots X_{d}} q_{i}^{c^{\prime}, t}}{X_{1} \ldots X_{d} T}\right) .
\end{aligned}
$$

Our cell-centric input entropy is defined by approximating $q_{i}^{c, t}$ by its average over all cells in the block whenever convenient. That is, we use the approximation

$$
\frac{\sum_{c^{\prime}=1}^{X_{1} \ldots X_{d}} q_{i}^{c^{\prime}, t}}{X_{1} \ldots X_{d}} \approx q_{i}^{c, t}
$$

in the argument to the logarithm in (10) for all $c$ such that $1 \leq c \leq X_{1} \ldots X_{d}$. We then obtain

$$
C_{f}^{t}\left(X_{1}, \ldots, X_{d}, T\right)=-\frac{1}{X_{1} \ldots X_{d}} \sum_{c=1}^{X_{1} \ldots X_{d}} \sum_{i=1}^{s^{1+\delta}}\left(\frac{q_{i}^{c, t}}{T}\right) \log _{s}\left(\frac{q_{i}^{c, t}}{T}\right),
$$

which is the cell-centric input entropy of the $X_{1} \times \cdots \times X_{d} \times T$ block that ends at time $t$.

The essential question, of course, is whether the cell-centric input entropy defined in (12) still has discriminatory capabilities analogous to those of the input entropy, given that the two are related by the approximation in (11). The answer to this question is affirmative and is explored in Section [5] through the mean $\overline{C_{f}}\left(X_{1}, \ldots, X_{d}, T\right)$ and the variance $\sigma^{2}\left(C_{f}\left(X_{1}, \ldots, X_{d}, T\right)\right)$, defined analogously to (8) and (9), respectively.

Let us then consider how much communication must be directed towards the special processor $P$ during a parallel simulation of a cellular automaton. Clearly, a processor $Q \neq P$ lodging $X_{Q}$ cells can calculate its portion of the double summation in (12) for each $t$ (i.e., let $c$ range over its $X_{Q}$ cells) completely locally. If $N$ denotes the number of processors, then $P$ has to receive $O\left(N t_{+}\right)$ floating-point numbers for the entire simulation. In the limit of true massive parallelism, this becomes $O\left(X_{1} \ldots X_{d} t_{+}\right)$, which relates to the communications requirements of the original input entropy by a factor of $O(\delta)$ if we disregard any differences between sending integers and sending floating-point numbers. So using the cell-centric approximation to input entropy saves considerable amounts of communication in the current technological reality of $N \ll X_{1} \ldots X_{d}$ but makes little sense in the limit of true massive parallelism.

\subsection{Cell-centric transition entropy}

We perceive the functional form of (12) as being suggestive of a host of possible different criteria that may be experimented with when attempting to classify cellular-automaton update rules. One possibility that we have considered is the 
following. For a fixed cell $c$ inside the $X_{1} \times \cdots \times X_{d} \times T$ block that ends at time $t$, let $\tau^{c, t}$ denote the number of state transitions within the block that cause the state of the cell to change during the simulation. This quantity does not depend explicitly on the inputs to the cell, so when deriving the corresponding cell-centric entropy measure from (12), and considering that there are $T-1$ state transitions per cell within the block, we obtain

$$
T_{f}^{t}\left(X_{1}, \ldots, X_{d}, T\right)=-\frac{1}{X_{1} \ldots X_{d}} \sum_{c=1}^{X_{1} \ldots X_{d}}\left(\frac{\tau^{c, t}}{T-1}\right) \log _{s}\left(\frac{\tau^{c, t}}{T-1}\right) .
$$

We call the quantity in (13) the cell-centric transition entropy relative to the block that ends at time $t$. Naturally, it shares with the cell-centric input entropy all the relevant characteristics that have to do with the parallel simulation of cellular automata. In addition, as will become apparent in Section 5 it also offers interesting glimpses into the categorization of the underlying update rule when analyzed from the perspective of its mean $\overline{T_{f}}\left(X_{1}, \ldots, X_{d}, T\right)$ and its variance $\sigma^{2}\left(T_{f}\left(X_{1}, \ldots, X_{d}, T\right)\right)$, defined in analogy to (8) and (9), respectively.

\subsection{Upper bounds}

Upper bounds on the cell-centric input entropy of (12) and the cell-centric transition entropy of (13) can be established easily if we recall that entropies are maximized when all the mutually exclusive events at hand are ascribed the same probability. In the case of (12) this amounts to setting $q_{i}^{c, t} / T$ to $s^{-(1+\delta)}$ for all appropriate values of $i$ and $c$, which yields

$$
C_{f}^{t}\left(X_{1}, \ldots, X_{d}, T\right) \leq 1+\delta .
$$

The case of (13) is even simpler, as it suffices to recognize that $x \log _{s} x$ is maximized for $x=e^{-1}$ and to set $\tau^{c, t} /(T-1)$ to this value for all appropriate values of $c$, thus yielding

$$
T_{f}^{t}\left(X_{1}, \ldots, X_{d}, T\right) \leq \frac{e^{-1}}{\ln s} .
$$

But some of the results described in Section $[5$ are based on the so-called outer-totalistic update rules 9], that is, update rules whose outcomes depend not on the cell's individual state and those of its neighbors, but rather on the cell's state and the sum of its neighbors' states. For such update rules, and considering the $s=2$ case only, while the bound given by (15) is still correct and gives a value slightly above 0.53 , in the case of the cell-centric input entropy it no longer makes sense to assume a uniform probability distribution on all inputs for entropy maximization, and consequently (14) has to be revised. The correct level at which to assume the uniform distribution for entropy to be maximized is now the level at which inputs are grouped with one another according to the sum of the neighbor states that they comprise.

For $s=2$ the number of distinct such groups is $1+\delta$, each corresponding to one of the possible sum values, from 0 to $\delta$. The probability associated with 
each group is then $(1+\delta)^{-1}$, so each individual input is assumed to occur with probability $[(1+\delta) n(\sigma)]^{-1}$, where $n(\sigma)$ is the number of inputs whose states sum up to $\sigma$ with $0 \leq \sigma \leq \delta$. But $n(\sigma)=\left(\begin{array}{l}\delta \\ \sigma\end{array}\right)$, so from (12) we obtain

$$
C_{f}^{t}\left(X_{1}, \ldots, X_{d}, T\right) \leq \log _{2}(1+\delta)+\frac{1}{1+\delta} \sum_{\sigma=0}^{\delta} \log _{2}\left(\begin{array}{l}
\delta \\
\sigma
\end{array}\right) .
$$

\section{Computer experiments}

An infinite cellular automaton cannot be simulated in its entirety, nor can a portion of it be simulated for an indefinitely long number of time steps. One crucial first decision when planning such a simulation is which contiguous cells to observe along each of the $d$ dimensions and also the number of time steps $t_{+}$ during which to perform the simulation. Choosing a finite number of cells to observe poses the question of how to handle the boundaries of the observed region, since those boundaries affect the simulation but cannot be extended indefinitely. Adopting artificial cylindrical boundaries or feeding randomly picked values to the boundary cells at each time step of the simulation will not in principle do, since this would have direct impact on the assumed infinite and deterministic nature of the cellular automaton.

The solution, naturally, comes from first setting the value $t_{+}$of the number of steps during which the cellular automaton is to be observed in the simulation, as well as the number $\ell_{k}$ of contiguous cells to be observed along the $k$ th dimension, $1 \leq k \leq d$, and then working backwards from them. We start by assuming a von Neumann neighborhood and then split a cell's neighborhood $\delta$ into its dimension-wise constituents; that is, we write $\delta=2\left(r_{1}+\cdots+r_{d}\right)$, where each $r_{k}$ is the update rule's radius along the $k$ th dimension. In order to output the state at time $t_{+}$of a cell that lies at a boundary along the $k$ th dimension as if it were indeed embedded in an infinite cellular automaton, the states of additional $r_{k}$ off-boundary cells are needed along that dimension at time $t_{+}-1$. The number of boundary cells along the $k$ th dimension at time $t_{+}$is

$$
2 \prod_{\substack{l=1 \\ l \neq k}}^{d} \ell_{l}
$$

so the total number of cells for which states are needed at time $t_{+}-1$ is

$$
\ell_{1} \ldots \ell_{d}+2 \sum_{k=1}^{d} r_{k} \prod_{\substack{l=1 \\ l \neq k}}^{d} \ell_{l} \leq \prod_{k=1}^{d}\left(\ell_{k}+2 r_{k}\right) .
$$

Note that, for $d=1$, equality holds in (18). The upper bound is useful, though, because it generalizes easily as we work backwards through time $t=0$, revealing that initial states are needed for cells that number no more than

$$
\prod_{k=1}^{d}\left(\ell_{k}+2 r_{k} t_{+}\right) \text {. }
$$


The upper bound in (19) is clearly an exaggeration for $d>1$ under a von Neumann neighborhood, since it corresponds to an $\left(\ell_{1}+2 r_{1} t_{+}\right) \times \cdots \times\left(\ell_{d}+\right.$ $\left.2 r_{d} t_{+}\right)$patch of cells. Here we only point out that specifying the precise cells whose initial states are needed is totally feasible, however cumbersome their determination or the inner mechanics of a parallel simulation involving exactly those cells and no others. In any event, we now have a set of cells that can be simulated through time $t_{+}$with the certainty that the observed behavior of the core $\ell_{1} \ldots \ell_{d}$ cells is fully compatible with the assumption of an infinite cellular automaton and of the deterministic character of its update rule. Boundaries still exist with respect to the extended set of cells, but the way they are handled is now immaterial. Either cylindrical boundaries may be assumed or randomly picked states may be used to fill up the inputs needed by the boundary cells. The effects of either choice can only affect the states of the core cells after time $t_{+}$.

When we assume a Moore neighborhood to start with, we write $\delta=(1+$ $\left.2 r_{1}\right) \ldots\left(1+2 r_{d}\right)-1$ instead, so that the number of cells for which states are needed at time $t_{+}-1$ is exactly the upper bound appearing in (18). In this case, clearly the number of cells given by (19) is no longer an exaggeration, but expresses precisely what is needed.

In Figure 1 we provide an illustration of these issues in the two-dimensional case when $\ell_{1}=3$ and $\ell_{2}=4$ with $r_{1}=2$ and $r_{2}=1$. What is shown is the set of cells for which initial states are needed if the states of the shaded cells are to be observed for $t_{+}=3$ further time steps as if those cells were part of an infinite, deterministic cellular automaton. Cells enclosed within the thick solid contour are those for which initial states are needed in the case of a von Neumann neighborhood. Those enclosed with the thick dashed contour must have initial states specified if a Moore neighborhood is used. Our practice henceforth is to employ sets of cells of the latter type regardless of the neighborhood type in use.

\subsection{The value of $T$}

When a cellular automaton is simulated with the goal of computing the input entropy of Section 3 or one of the cell-centric quantities of Section 4 inside an $X_{1} \times \cdots \times X_{d} \times T$ state block, the core set of observed cells is such that $\ell_{1}=X_{1}, \ldots, \ell_{d}=X_{d}$. In this section, we discuss the choice of $T$ that maximizes the discriminatory capabilities of our cell-centric heuristics in the context of the Wolfram classes. We henceforth assume $S=\{0,1\}$, i.e., $s=2$.

Our approach has been to perform a set of initial experiments with $t_{+}=$ 500 on a single processor and to analyze their outcomes aiming at finding a suitable $T$ value for use in the main experiments. We ran four sets of initial experiments: one for $d=1$ and $r_{1}=2$, one for $d=1$ and $r_{1}=3$, one for $d=2$ under a von Neumann neighborhood with $r_{1}=r_{2}=1$, and one last for $d=2$ under a Moore neighborhood with $r_{1}=r_{2}=1$. For the one-dimensional cases, each set comprised four runs for $X_{1}=150$ and four runs for $X_{1}=300$; for the two-dimensional cases, we did four runs for each of $X_{1}=X_{2}=15$ and 


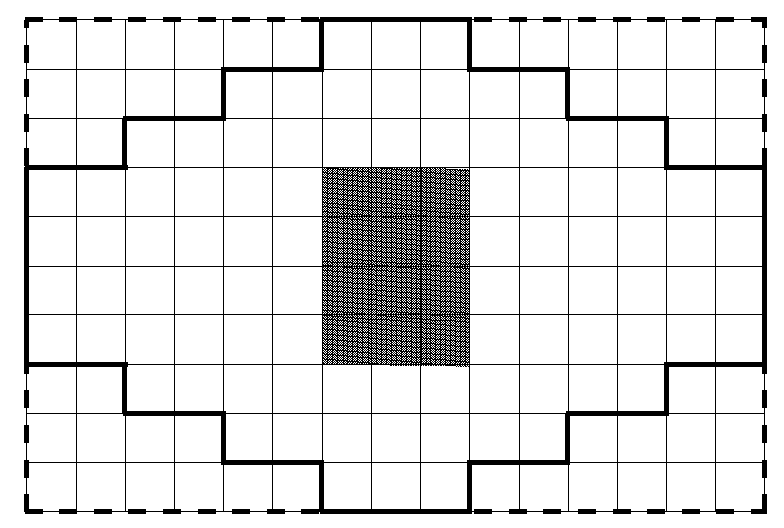

Figure 1: The $15 \times 10$ patch of cells for which initial states are needed in the two-dimensional case of $\ell_{1}=3, \ell_{2}=4, r_{1}=2$, and $r_{2}=1$, so that the shaded cells may be observed correctly for $t_{+}=3$. The thick-line enclosures refer to the minimal sets of cells that are needed under a von Neumann (solid line) or a Moore (dashed line) neighborhood.

$X_{1}=X_{2}=30$. Within each set, the first run corresponds to a known class-(i) update rule, the second to a known class-(ii) update rule, and so on. The known update rules we used are detailed in Table 1

In Table 1 the update rules are specified according to the following conventions. For the one-dimensional experiments, each update rule is the hexadecimal form of the binary number whose most significant bit is the update rule's output to the input $11 \ldots 1$, read left to right, the next bit corresponds to $11 \ldots 1-1$, and so on (cf., e.g., 22]). The two-dimensional von Neumann case is similar, except that the most significant bit corresponds to $00 \ldots 0$, the next one to $00 \ldots 0+1$, and so on, inputs being read in the self-north-east-south-west order 23]. The two-dimensional Moore case comprises outer-totalistic update rules only, for which we adopt Conway's Life 24, 25] usual notation style: bx indicates that the cell's state moves from 0 to 1 (it "is born") if the cell has $x$ neighbors in the 1 state; $\mathbf{s} x$ means that the cell's state remains 1 (it "survives") if the cell has $x$ neighbors in the 1 state; in all cases not listed explicitly, the cell's state becomes 0 [23.

Within each run, the cellular automaton is simulated for $T=5,10, \ldots, 250$ and for each simulation the mean and variance indicators of Section 4 are computed. Simplifying the notation in the obvious way, these are $\overline{C_{f}}, \sigma^{2}\left(C_{f}\right), \overline{T_{f}}$, and $\sigma^{2}\left(T_{f}\right), f$ being the update rule under consideration. All simulations sharing the same value of $d, X_{1}, \ldots, X_{d}$, and $r_{1}, \ldots, r_{d}$ start at the same initial configuration, itself generated at the beginning for that group of simulations 
Table 1: Update rules used to generate the plots in Figures 20 5

\begin{tabular}{|c|c|c|}
\hline Experiment & Class & Update rule \\
\hline$d=1$ & (i) & $1 \mathrm{~d} 000 \mathrm{a} 20$ \\
\hline$r_{1}=2$, & (ii) & $01 \mathrm{dc} 3610$ \\
\hline \multirow[t]{2}{*}{ Figures $2(\mathrm{a}-\mathrm{d})$ and $4(\mathrm{a}-\mathrm{d})$} & (iii) & $994 a 6 a 65$ \\
\hline & (iv) & $6 c 1 e 53 a 8$ \\
\hline$d=1$ & (i) & $1 \mathrm{df} 00000000 \mathrm{f} 00000000000000000020$ \\
\hline$r_{1}=3$, & (ii) & $7 \mathrm{fdc} 3610 \mathrm{f}$ c48472c01dc361001dc3660 \\
\hline \multirow[t]{2}{*}{ Figures $2(\mathrm{e}-\mathrm{h})$ and $4(\mathrm{e}-\mathrm{h})$} & (iii) & $994 f 6 a 65994 a 6 a 65 a 94 a 6 a 65994 a 6 a 99$ \\
\hline & (iv) & $3 b 469 c 0 e e 4 f 7 f a 96 f 93 b 4 d 32 b 09 e d 0 e 0$ \\
\hline$d=2$, & (i) & 00000601 \\
\hline$r_{1}=r_{2}=1$ & (ii) & 06900600 \\
\hline von Neumann, & (iii) & 69969669, Fredkin2 23] \\
\hline Figures $3(\mathrm{a}-\mathrm{d})$ and $5(\mathrm{a}-\mathrm{d})$ & (iv) & 6db6fac8, Crystal2 [23] \\
\hline$d=2$ & (i) & b3b6b7 s3s6s7s8 \\
\hline$r_{1}=r_{2}=1$ & (ii) & b3 s2s5s6 \\
\hline Moore, & (iii) & b1b3b5 s1s3s5 \\
\hline Figures $3(\mathrm{e}-\mathrm{h})$ and $5(\mathrm{~s}-\mathrm{h})$ & (iv) & b3 s2s3 \\
\hline
\end{tabular}

by randomly choosing initial states for the $\left(X_{1}+2 r_{1} t_{+}\right) \ldots\left(X_{d}+2 r_{d} t_{+}\right)$cells involved.

The results of these initial experiments are given in Figures 2 through 5 and also in Appendix $\mathrm{A}$ where plots of spatiotemporal patterns are given for selected runs. Figures 2 and 3 refer, respectively, to the behavior of the cell-centric input entropy for the one- and two-dimensional cases as $T$ varies. Figures 4 and 5 . in turn, refer to the behavior of the cell-centric transition entropy for the oneand two-dimensional cases, respectively, as $T$ varies. Notice that, but virtue of our experiments' setup, the plots in Figures 2 and 4 for which $r_{1}$ and $X_{1}$ have the same values correspond to the same initial configuration, and similarly for Figures 3 and 5

Even though this first set of experiments is deprived of statistical significance (based as it is on runs from a single initial configuration), it provides an initial indication of the discriminatory capabilities of our cell-centric heuristics. In fact, an examination of all mean- and variance-plot pairs in Figures 25 reveals that, with a few exceptions, classes (i)-(iv) can in the worst case be discriminated within roughly one order of magnitude by either the mean or the variance of both the cell-centric input and transition entropies for most values of $T$. For example, comparing the plots in Figures 2 (a) and (b) indicates that the mean cell-centric input entropy provides good discrimination among the four classes, except between classes (iii) and (iv), which nonetheless can be told apart easily by the variance of that entropy. The exceptions are the two-dimensional cases 

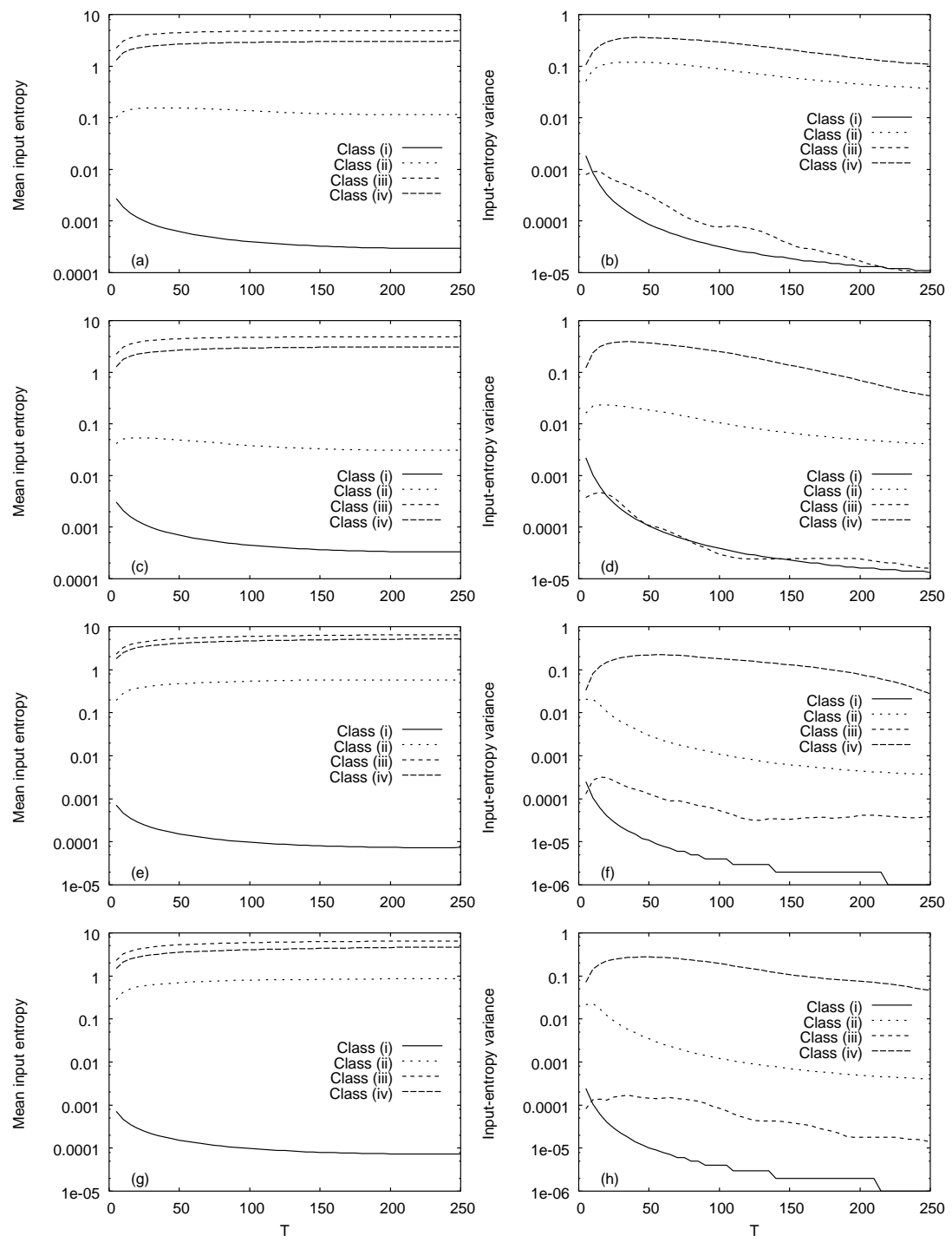

Figure 2: Mean $\left(\overline{C_{f}}\right)$ and variance $\left(\sigma^{2}\left(C_{f}\right)\right)$ of the cell-centric input entropy as a function of $T$ under four different update rules, one from each of classes (i) through (iv), for $d=1$. Data are given for the 150-cell case with $r_{1}=2$ (a and b), the 300-cell case with $r_{1}=2$ (c and d), the 150-cell case with $r_{1}=3$ (e and f), and the 300-cell case with $r_{1}=3$ (g and h). 

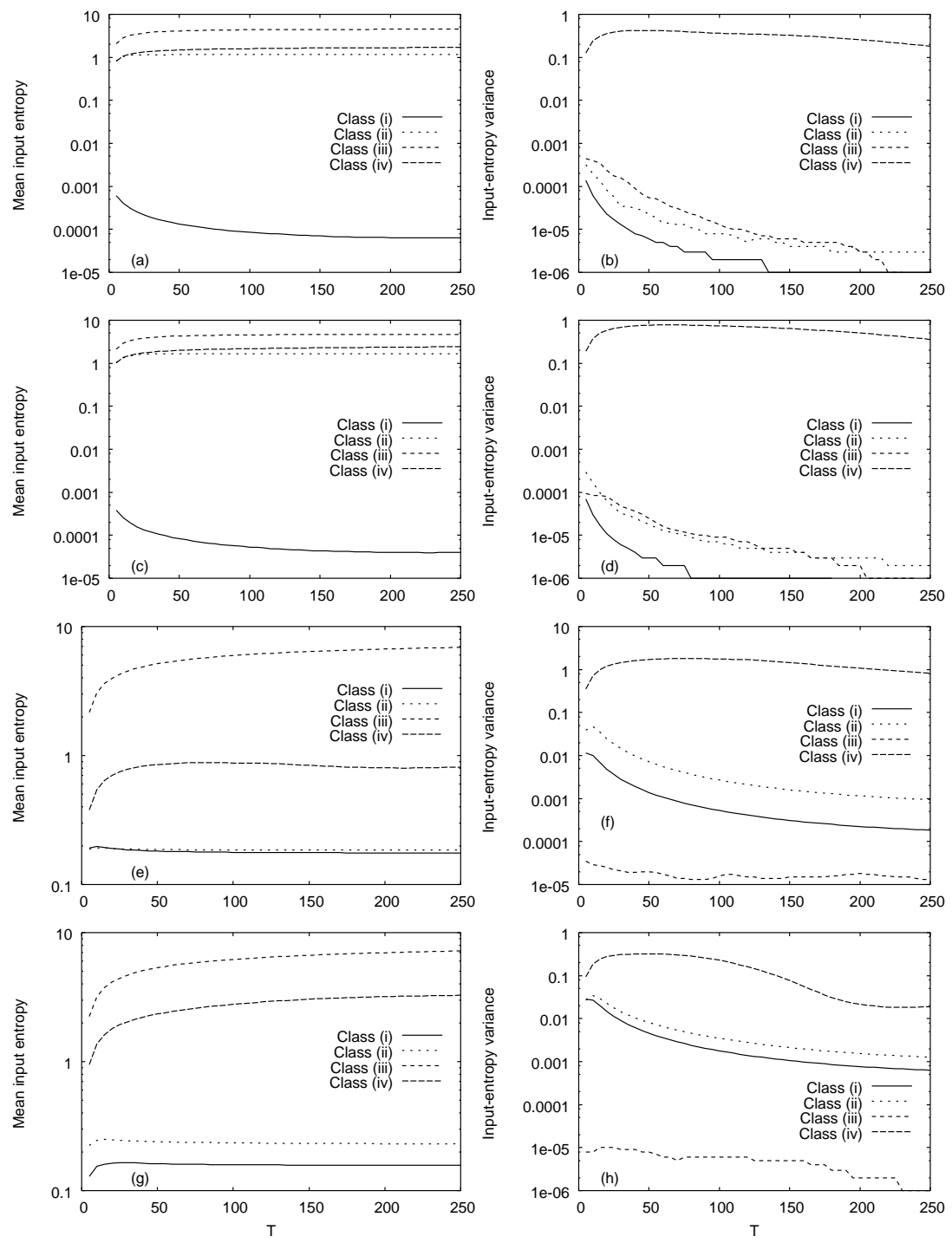

Figure 3: Mean $\left(\overline{C_{f}}\right)$ and variance $\left(\sigma^{2}\left(C_{f}\right)\right)$ of the cell-centric input entropy as a function of $T$ under four different update rules, one from each of classes (i) through (iv), for $d=2$. Data are given for the $(15 \times 15)$-cell von Neumann case $(\mathrm{a}$ and $\mathrm{b})$, the $(30 \times 30)$-cell von Neumann case $(\mathrm{c}$ and $\mathrm{d})$, the $(15 \times 15)$-cell Moore case $(\mathrm{e}$ and $\mathrm{f})$, and the $(30 \times 30)$-cell Moore case $(\mathrm{g}$ and $\mathrm{h})$. In all cases, $r_{1}=r_{2}=1$. 

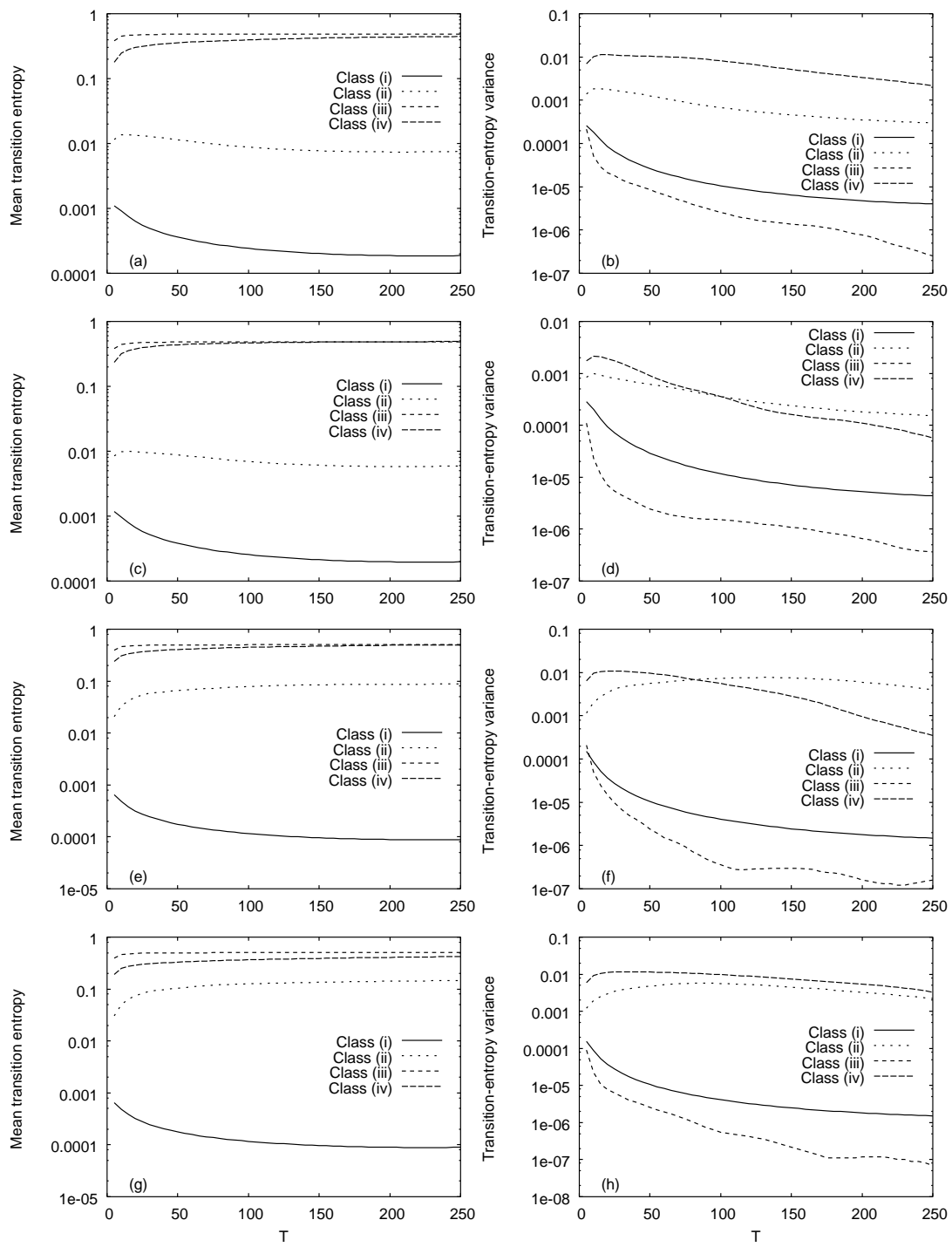

Figure 4: Mean $\left(\overline{T_{f}}\right)$ and variance $\left(\sigma^{2}\left(T_{f}\right)\right)$ of the cell-centric transition entropy as a function of $T$ under four different update rules, one from each of classes (i) through (iv), for $d=1$. Data are given for the 150-cell case with $r_{1}=2$ (a and b), the 300-cell case with $r_{1}=2$ (c and d), the 150-cell case with $r_{1}=3$ (e and f), and the 300-cell case with $r_{1}=3$ (g and h). 

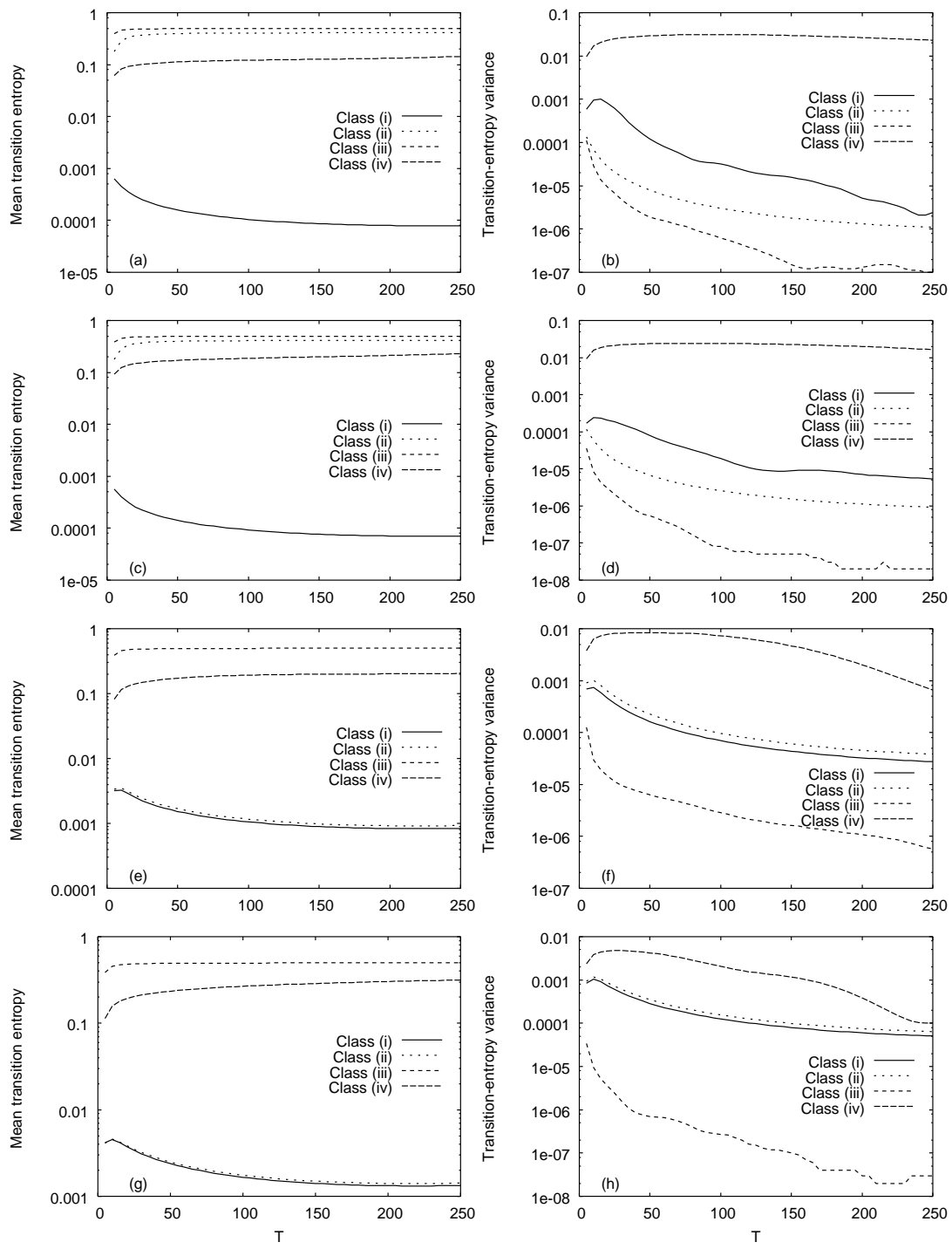

Figure 5: Mean $\left(\overline{T_{f}}\right)$ and variance $\left(\sigma^{2}\left(T_{f}\right)\right)$ of the cell-centric transition entropy as a function of $T$ under four different update rules, one from each of classes (i) through (iv), for $d=2$. Data are given for the $(15 \times 15)$-cell von Neumann case $(\mathrm{a}$ and $\mathrm{b})$, the $(30 \times 30)$-cell von Neumann case $(\mathrm{c}$ and $\mathrm{d})$, the $(15 \times 15)$-cell Moore case $(\mathrm{e}$ and $\mathrm{f})$, and the $(30 \times 30)$-cell Moore case $(\mathrm{g}$ and $\mathrm{h})$. In all cases, $r_{1}=r_{2}=1$. 
with Moore neighborhoods, in which neither of the cell-centric heuristics seems to be able to capture the distinction between classes (i) and (ii).

We return to this discussion of our heuristics' discriminatory capabilities shortly, after we have provided more significant data. But returning to the original goal of this initial set of experiments, we see from the plots in Figures 25 that several possibilities exist for choosing a value for $T$. We note that, naturally, choosing as small a value as possible has the advantage of alleviating the processing demands for computing the entropy figures. With these observations in mind, our choice hereafter is to use $T=25$.

\subsection{Experimental results}

Simulating a cellular automaton in parallel is essentially an exercise in designing a simple synchronous distributed algorithm, in the sense described in [26], employing for synchronization the technique of $\alpha$-synchronization of [27]. Within this general framework, several proposals have been put forward (cf., e.g., 28, 29, 30]).

Our parallel simulator is no exception and has been designed and implemented within this same framework for one- and two-dimensional cellular automata. Each simulation is initiated by partitioning the automaton into the $N$ available processors. The hardware we have used in all the experiments described henceforth has $N=8$. All processors have the capability of communicating directly with all others. For $d=1$, the cells are partitioned equitably among the processors in such a way that each processor receives a contiguous set of cells to simulate; for $d=2$, the automaton is subdivided into rectangles of contiguous cells by slicing it equitably along the dimension that has the least number of cells.

Notice that the neighborhood relation among cells as given by the lattice that underlies the cellular automaton automatically implies a neighborhood relation among processors, too. Specifically, two processors are neighbors whenever at least one cell that one of them lodges is a neighbor of a cell lodged by the other. Obviously, some of the cells that a processor lodges are distinguished in that their states are needed by the processor's neighbors; we refer to such cells as frontier cells.

The simulation proper starts at each processor with the assignment of a randomly chosen initial state to each of the cells it lodges and the sending of the initial states of all frontier cells to the neighbor processors at which they are needed. The processor then iterates as $t$ is incremented from 0 through $t_{+}$: for each $t$, new states are computed for all the cells that the processor lodges and so are the portions of (12) and (13) corresponding to those of its cells that are observed, provided $t \geq T-1$; then the new states of the processor's frontier cells are sent where they are needed. At the end, each processor that lodges at least one observed cell forwards its $2\left(t_{+}-T+2\right)$ entropy results to a previously designated processor for computation of the two means and variances (viz. the means $\overline{C_{f}}$ and $\overline{T_{f}}$ and the variances $\sigma^{2}\left(C_{f}\right)$ and $\left.\sigma^{2}\left(T_{f}\right)\right)$. 


\section{One-dimensional cellular automata}

Our setup for the one-dimensional experiments is based on either $r_{1}=2$ or $r_{1}=3$. For $r_{1}=2$, the setup has $X_{1}=2000, t_{+}=500$, and $T=25$. The overall number of cells to simulate is then $X_{1}+2 r_{1} t_{+}=4000$, so the number of observed cells constitutes half of the total. For $r_{1}=3$, our setup has $X_{1}=2400$, $t_{+}=400$, and $T=25$. In this case, the total number of cells in the simulation is 4800 , and once again the observed cells account for half the total number of cells.

In the one-dimensional case, the number of distinct update rules is given by $2^{2^{1+2 r_{1}}}$, that is, $2^{32}$ for $r_{1}=2$ and $2^{128}$ for $r_{1}=3$. Our results are based on 50000 update rules randomly chosen out of those and are shown in Figure 6 as plots of the variances $\sigma^{2}\left(C_{f}\right)$ and $\sigma^{2}\left(T_{f}\right)$ against the means $\overline{C_{f}}$ and $\overline{T_{f}}$, respectively. The points that correspond to the one-dimensional update rules of Table 1 are not shown explicitly but are singled out by indications, at their coordinates, of the classes to which the update rules belong.

One crucial information that has been left out of the plots in Figure [in order to avoid any further cluttering is the density of points at any particular meanvariance region. We provide some of this information next. First we choose, for each of the plots, a value for the mean entropy that separates the update rules labeled (i) or (ii) from those labeled (iii) or (iv). In Figures [ (a) and (b), which refer to the cell-centric input entropy, this mean entropy can be taken to be 1 ; in Figures [ (c) and (d), it can be taken to be 0.1. Selecting this value partitions the plot into two regions and for each one we now select an entropy variance that can be used to separate the update rules labeled (i) and (ii) on the left, and another that can likewise be used for those labeled (iii) and (iv). In parts (a) and (b) of the figure, our choices are 0.001 and 0.1 , respectively on the left and right sides; in parts (c) and (d) the corresponding values are 0.0001 and 0.001. At the end, in each plot we are left with a partition into four regions, each containing exactly one of the update rules labeled (i)-(iv).

We may then provide the missing information. In part (a), $2.30 \%$ of the update rules are inside the (i) region, $8.10 \%$ in the (ii) region, $86,96 \%$ in the (iii) region, and $2.64 \%$ in the (iv) region. Part (b) contains no update rules inside the (i) region, $0.80 \%$ of the update rules in region (ii), $97.85 \%$ in (iii), and $1.35 \%$ in (iv). In part (c) we have the figures $2.54 \%, 9.30 \%, 83.47 \%$, and $4.69 \%$. In part (d) we once again have no update rules inside the (i) region and the remaining figures are $0.80 \%, 97.59 \%$, and $1.61 \%$. The well-known preponderance of class-(iii) update rules, as well as the relative rarity of class-(iv) update rules, particularly as $r_{1}$ is increased from parts (a) and (c) to parts (b) and (d), are then confirmed.

\section{Two-dimensional cellular automata}

For the two-dimensional experiments we use $r_{1}=r_{2}=1$ throughout. Regardless of the neighborhood type (von Neumann or Moore), our experiments' setup has $X_{1}=X_{2}=100, t_{+}=50$, and $T=25$. The total number of cells to be simulated 

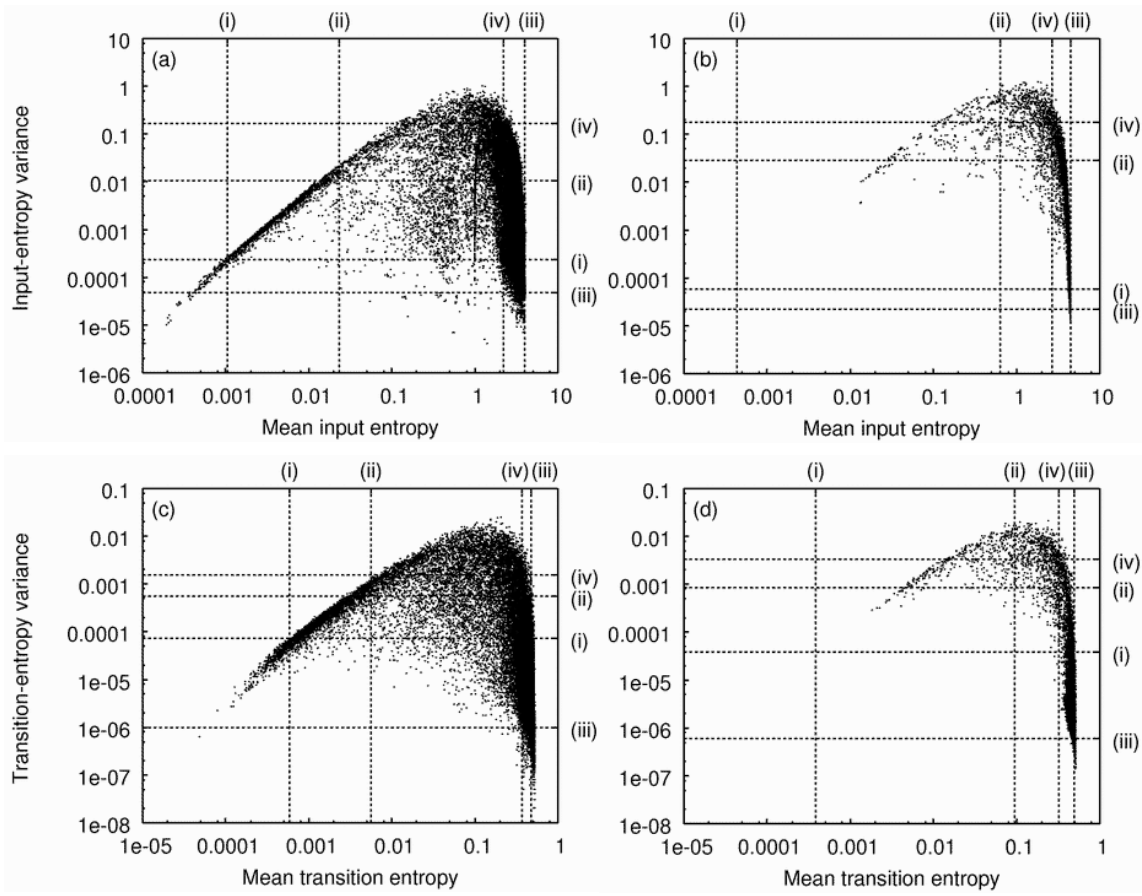

Figure 6: Occurrence of mean-variance pairs within the general experimental setup for one-dimensional cellular automata. Data are given for the cell-centric input entropy with $r_{1}=2$ (a) and $r_{1}=3$ (b) as plots of $\sigma^{2}\left(C_{f}\right)$ against $\overline{C_{f}}$, and also for the cell-centric transition entropy with $r_{1}=2$ (c) and $r_{1}=3$ (d) as plots of $\sigma^{2}\left(T_{f}\right)$ against $\overline{T_{f}}$. Each plot contains 50000 points, each point corresponding to a randomly chosen update rule and to an average over 5 randomly chosen initial configurations. The one-dimensional update rules of Table 1 are also shown within the same experimental setup, but not as points: instead, they are singled out with an indication at their coordinates of which of classes (i)-(iv) they belong to. 
is therefore $\left(X_{1}+2 r_{1} t_{+}\right)\left(X_{2}+2 r_{2} t_{+}\right)=40000$, so the number of observed cells is one quarter of the total number.

Similarly to the one-dimensional case, in the two-dimensional case with von Neumann neighborhoods there are $2^{2^{1+2\left(r_{1}+r_{2}\right)}}=2^{32}$ distinct update rules. With Moore neighborhoods, and considering only outer-totalistic update rules, the number of distinct update rules is $2^{2\left(1+2 r_{1}\right)\left(1+2 r_{2}\right)}=2^{18}$. Once again, in both cases our results are based on 50000 update rules randomly chosen out of the corresponding sets. ${ }^{1}$ They are shown in the plots of Figure 7 in the same style as Figure [6] As in the case of that figure, the marginal indications (i)-(iv) give the coordinates at which the points corresponding to the two-dimensional update rules of Table 1 would be found, had they been plotted explicitly. Parts (a) and (c) of the figure refer to a von Neumann neighborhood, parts (b) and (d) to a Moore neighborhood.

Once again additional information regarding the density of points in the four plots must be given on the side. Following the same methodological steps as for the one-dimensional cases, first we select a mean-entropy value for each plot to separate the update rules labeled (i) or (ii) from those labeled (iii) or (iv), and then we select an entropy-variance value to separate each pair of labeled update rules. As the four plots in Figure [7 indicate, this may prove a harder task than in the one-dimensional cases, since it is now common to find two or more labels clustered together along one of the axes.

Let us begin with part (a), which refers to the cell-centric input entropy under a von Neumann neighborhood. If we select 1.73 as the first separator, and then select 0.0005 on the left and 0.1 on the right, then we are left with no update rules lying within class-(i) region, while $7.92 \%$ of the update rules are in region (ii), $90.47 \%$ in region (iii), and $1.61 \%$ in region (iv). Moving to part (b) we select the separators $1,0.007$, and 0.1 , which yields the percentages $4.42 \%, 12.08 \%$, $77.19 \%$, and $6.31 \%$, respectively for regions (i)-(iv), relative to the cell-centric input entropy under a Moore neighborhood. The remaining two plots, in parts (c) and (d), are both relative to the cell-centric transition entropy, respectively under a von Neumann and a Moore neighborhood. Selecting the separators 0.4, 0.0002 , and 0.0001 in the former case yields $6.67 \%, 16.07 \%, 73.27 \%$, and $3.98 \%$. In the latter case, we select $0.1,0.0072$, and 0.0001 , obtaining $19.02 \%, 1.82 \%$, $70.74 \%$, and $8.42 \%$. As in the one-dimensional case, indications are once again clear concerning the relative predominance and rarity of classes (iii) and (iv), respectively.

\footnotetext{
${ }^{1}$ Note that restricting Moore-neighborhood update rules to lie within the set of outertotalistic update rules is a means to ensure that these 50000 samples have some statistical representativeness. In the absence of this restriction, the number of possible update rules becomes $2^{2^{\left(1+2 r_{1}\right)\left(1+2 r_{2}\right)}}$. This number, with values for $r_{1}$ and $r_{2}$ as we have adopted, is $2^{512}$, which is larger by more than a hundred orders of magnitude than the number of distinct update rules in any of our other experiments.
} 

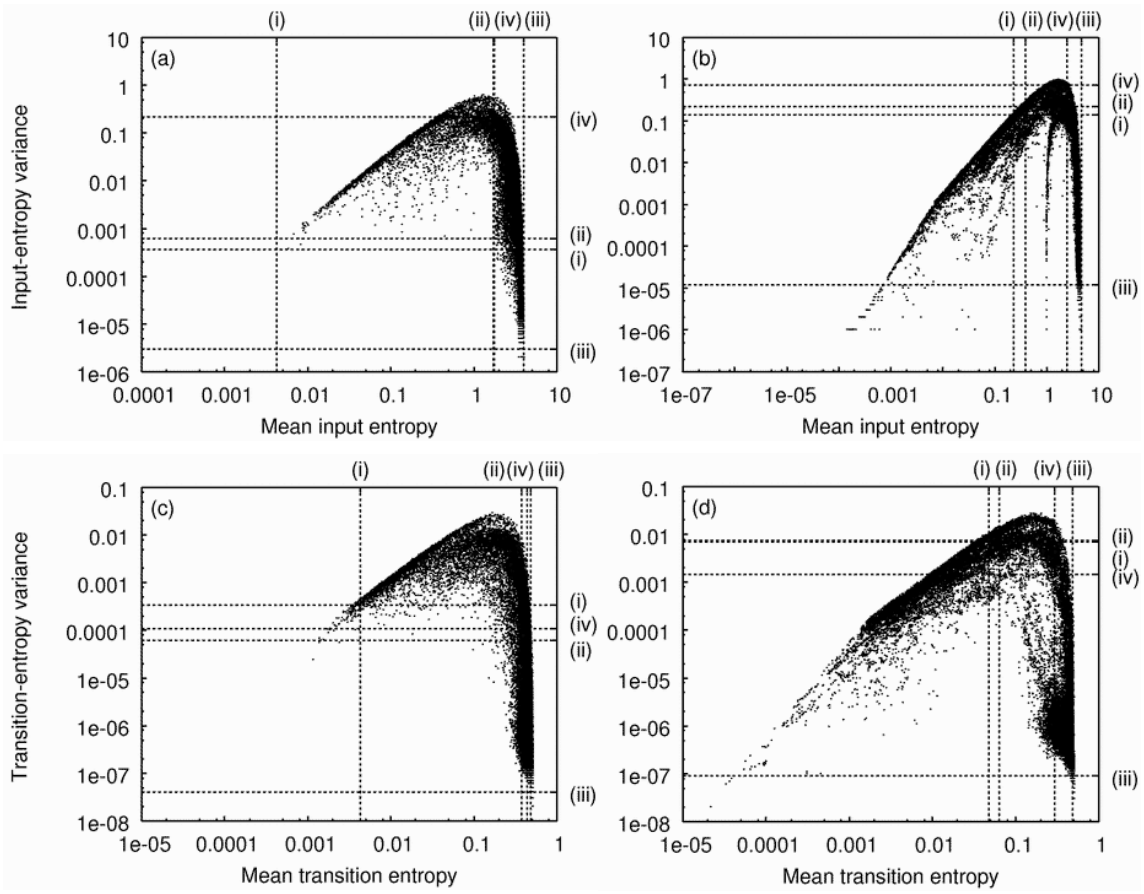

Figure 7: Occurrence of mean-variance pairs within the general experimental setup for two-dimensional cellular automata. Data are given for the cell-centric input entropy with the von Neumann (a) and the Moore (b) neighborhoods as plots of $\sigma^{2}\left(C_{f}\right)$ against $\overline{C_{f}}$, and also for the cell-centric transition entropy with the von Neumann (c) and the Moore (d) neighborhoods as plots of $\sigma^{2}\left(T_{f}\right)$ against $\overline{T_{f}}$. Each plot contains 50000 points, each point corresponding to a randomly chosen update rule and to an average over 5 randomly chosen initial configurations. The two-dimensional update rules of Table 1 are also shown within the same experimental setup, but not as points: instead, they are singled out with an indication at their coordinates of which of classes (i)-(iv) they belong to. 


\section{Discussion}

The data shown in Figures 6 and 7 respectively for one- and two-dimensional cellular automata, tend all to exhibit the following behavior regarding classes (i)-(iv). When plotted on doubly-logarithmic scales, they appear clustered roughly as a boomerang whose traversal from the low-mean, low-variance tip leads us through class-(i) update rules, then class (ii), then class (iv) near the middle bend, and finally class (iii) past the bend. Entropy means increase at varying rates along the traversal, while variances increase at first but fall down again after the middle bend in the cluster's shape.

The one-dimensional cases, depicted in Figure [6] indicate unequivocally that the eight one-dimensional update rules of Table 1 can be told apart by at least one full order of magnitude of the mean entropy or the entropy variance, often both, regardless of which cell-centric entropy is being used. While the same holds unchanged for the case shown in Figure Z(a), which refers to the cellcentric input entropy under a von Neumann neighborhood, the remaining three parts, (b)-(d), must be examined in more detail. The case of part (b), in which the input entropy is still the one in use but now under a Moore neighborhood, allows proper separation between classes (iii) and (iv), but apparently leave classes (i) and (ii) mixed up together. The picture as we move to part (c), which corresponds to the cell-centric transition entropy under a von Neumann neighborhood, is once again subject to mix-ups, this time between classes (ii) and (iv). The final case is that of part (d), corresponding to the transition entropy and to a Moore neighborhood. In this case, as in the case of part (b), classes (i) and (ii) are hard to tell apart.

We envisage two major trends underlying these class mixtures in the meanvariance plots for the two-dimensional cases. The first one has to do with part (c) of Figure 7 where a mix-up of classes (ii) and (iv) under a von Neumann neighborhood turns up when the transition entropy is used. What may be happening is that the relatively low value of 50 chosen for $t_{+}$(for the strictly practical reason of keeping our run times within reasonable bounds while the plots of the figure were produced) is insufficient for the automaton to settle into a more typical class-(ii) behavior (and hence mean and variance values of the transition entropy that are commensurate with that class). This is somewhat supported by an examination of Figure 9 but clearly calls for additional investigation (more on this in Section 7 ).

The second trend concerns parts (b) and (d) of Figure 7 mainly, and thus has to do with the mix-up between classes (i) and (ii) when a Moore neighborhood is in use, but may also be related to the case of part (c) we just discussed. Our expectation that the Wolfram classification carries on naturally to the twodimensional case comes from Wolfram's own investigations on two-dimensional cellular automata 9, but this has been challenged on the grounds that such a classification scheme fails to recognize the real sign of complex behavior in outertotalistic, two-dimensional update rules, which is the presence of the so-called gliders, that is, the structures that are seen to "glide" across the two-dimensional 
lattice as time elapses 31, 32..$^{2}$ If this is the case, then what is out of place is not the mix-up of classes (i) and (ii), but rather the separation between the two classes, since none of the two exhibits gliders and should therefore be coalesced together into one single class.

But beyond these slight conflicts, and whichever of the competing trends may win at the end, we perceive our experiments' outcomes as expressed in Figures 6 and 7 as laying out an overall methodology for the classification of cellularautomaton update rules, one that in many senses confirms the initial conclusions of [21. First and foremost is an examination of the mean entropies vis-à-vis the bounds made available in (14) through (16). For the one-dimensional cases, (14) predicts that no mean cell-centric input entropy goes beyond $1+2 r_{1}$, while predicting $1+2\left(r_{1}+r_{2}\right)$ as the maximum for the von Neumann two-dimensional case. Thus, the upper bound turns out to be 5 in the case of part (a) of Figure 6] 7 for part (b), and 5 for Figure 7(a). The tighter number given by (16) for outer-totalistic, Moore-neighborhood update rules yields approximately 6.87 for Figure 7(b). While the four plots respect the corresponding upper bounds (this may not be immediate from the figures, owing to the logarithmic scale, but we know it from our files and refrain from presenting further plots), none of the 50000 randomly chosen update rules comes very near its bound. Perhaps this is due to the difficulty of sampling an update rule whose mean input entropy comes sufficiently near the bound, but the fact remains that comparing an update-rule's mean input entropy to its known upper bound may be of little help towards classifying the update rule.

The case of parts (c) and (d) of both Figures [6] and 7 being as they are based on the cell-centric transition entropy, is different. In this case, meeting the upper bound of approximately 0.53 given by (15) does not seem to depend on serendipitously finding any particular update rule. In fact, update rules whose mean transition entropy approach the bound closely occur frequently, as once again can be seen in the figures. When using the transition entropy, then, a useful first step is to compare the update rule's mean entropy with this bound: if close enough, almost surely the update rule is a class-(iii) one.

Beyond this initial test against known upper bounds, what remains of the aforementioned overall methodology is essentially a cladistics-like ${ }^{3}$ buildup of relationships among update rules given their cell-centric (input and/or transition) entropy means and variances. The crux here is that classification is the product of comparison, thence the fundamental importance of update rules such as the ones in Table 1 for which we are capable of providing a desired classification a priori so they can function as seeds in the larger classification process.

\footnotetext{
${ }^{2}$ More generally, there have been arguments calling for classification schemes that take the particular application area under study into consideration more seriously (cf., e.g., 15).

${ }^{3}$ Here we allude to the method known as cladistics for hypothesizing relationships among (extant or extinct) organisms. Beyond its core assumptions, the method in essence relies on examining several characters of the organisms and employing them for grouping the organisms into the desired taxa [33.
} 


\section{Conclusions}

We have in this paper addressed the automatic classification of the update rules of cellular automata. Our departing point has been the notion of input entropy, on which we built by the introduction of two novel entropy measures, both inspired by, and targeted at, the simulation of cellular automata by messagepassing parallel machines. Our two new measures are the cell-centric input entropy and the cell-centric transition entropy. For both of them we provided extensive experimental results on both one- and two-dimensional cellular automata. Within our assumed classification context, that of Wolfram's four-class scheme, these results demonstrated that the two new measures provide satisfactory discriminatory capabilities in the one-dimensional case, while in the twodimensional case it is also a good discriminator but in addition helps support other authors' suggestions that a better classification scheme may be needed.

Our experimental results were the product of a parallel implementation of a simulator coupled with a module for calculating the two cell-centric entropies. We finalize by commenting on some performance-related aspects of this simulator. The results presented in Section 5 were obtained on an eight-computer cluster, each based on an Intel Pentium 4 processor running at $1.8 \mathrm{GHz}$ and having 1 gigabyte of memory. The eight computers are fully interconnected by a gigabit-ethernet switch. On this cluster, each of the eight test suites of Section [5] comprising 5 independent runs for each of 50000 update rules, requires somewhere from three to six days to complete, depending on which of the four update-rule categories (one-dimensional with two possible radii, von Neumann two-dimensional, Moore two-dimensional) and which of the cell-centric measures (input or transition entropy) are being used.

The fact that we are simulating infinite cellular-automata, as explained right at the beginning of Section [5 is naturally the source of considerable load imbalance among the processors. We have paid no heed to this issue, but clearly

it has to be reckoned with by anyone undertaking the parallel simulation of large-scale cellular automata if the effects of infinite boundaries are to be taken into account. There are two kinds of load imbalance to be considered. First is the fact that only those processors that lodge some of the observed cells do actually perform entropy-related calculations; among these, those that lodge more of those cells are more loaded by that kind of computation. Secondly, cells that are not observed but do nonetheless participate in the simulation for the sole sake of providing the illusion of an infinite cellular automaton do not have to be simulated for all the $t_{+}$steps; instead, as time elapses progressively less of such cells need to be simulated. Once these two types of load imbalance are taken into account, there are all sorts of policies that can be adopted to re-balance the computational load among the processors. We dwell on the issue no further in this paper, but it is clearly important and should be considered upon embarking in a more performance-aware implementation.

Another important aspect that ultimately is closely related to these performance issues is whether the need really exists to undertake the simulation of cellular automata with all the extra load for providing the illusion of infin- 
ity. While unquestionably this seems the right way to approach the simulation when a new classification scheme is first being tested, perhaps once it is established the infinity requirement may be dropped and cylindrical boundary conditions adopted instead. We have performed a few experiments with this trade-off in mind; their outcomes are shown in Table 2. Examining the table carefully reveals clearly that both of our cell-centric heuristics retain the same discriminatory capabilities we found them to possess in Section 5 even though occasionally the relative positioning of the classes with respect to the mean or variance of some entropy may not be the same, possibly due to the different numbers of cells in the two sets of experiments. In fact, a quick examination of Figures 10 and 11] which depict the spatiotemporal patterns of some cellular automata with cylindrical boundaries, reveals the same features we have come to associate with classes (i)-(iv), despite the artificial periodicity that appears in some cases as a result of assuming finite boundaries. But, as demonstrated by the results in Table 2 such periodicity appears to have no noticeable effect on our cell-centric entropies. One immediate consequence of this is that considerably larger cellular automata can now be simulated with the same overall processing effort, and also that the sources of load imbalance we discussed earlier become moot. Likewise, the simulation of two-dimensional cellular automata for significantly larger values of $t_{+}$becomes more viable, which perhaps may lead to a clarification of the mix-up between classes (ii) and (iv) under a von Neumann neighborhood alluded to in Section 6 
Table 2: Means and variances averaged over 5 runs from randomly chosen initial configurations with cylindrical boundaries for $t_{+}=500$ and $T=25$. Experiment codes are as follows. I: $d=1,150$ cells, $r_{1}=2$; II: $d=1,300$ cells, $r_{1}=2$; III: $d=1,150$ cells, $r_{1}=3$; IV: $d=1$, 300 cells, $r_{1}=3$; V: $d=2$, von Neumann neighborhood, $15 \times 15$ cells, $r_{1}=r_{2}=1$; VI: $d=2$, von Neumann neighborhood, $30 \times 30$ cells, $r_{1}=r_{2}=1$; VII: $d=2$, Moore neighborhood, $15 \times 15$ cells, $r_{1}=r_{2}=1$; VIII: $d=2$, Moore neighborhood, $30 \times 30$ cells, $r_{1}=r_{2}=1$. Update rules are as given in Table 1 for each of classes (i)-(iv). Numbers are truncated to six decimal places.

\begin{tabular}{lcccc}
\hline \multirow{2}{*}{ Experiment } & \multicolumn{4}{c}{$\overline{C_{f}}$} \\
\cline { 2 - 5 } I & (i) & (ii) & (ii) & (iv) \\
\cline { 2 - 5 } II & 0.000419 & 0.004730 & 4.671842 & 2.726404 \\
III & 0.001030 & 0.023110 & 3.997883 & 2.396267 \\
IV & 0.000170 & 0.685005 & 5.757584 & 3.195973 \\
V & 0.000146 & 0.723408 & 5.755847 & 3.527819 \\
VI & 0.000214 & 1.434876 & 3.692984 & 1.854994 \\
VII & 0.000245 & 1.543048 & 3.843658 & 1.506388 \\
VIII & 0.066639 & 0.110987 & 4.268379 & 1.206355 \\
& 0.134897 & 0.148938 & 4.431762 & 1.452313 \\
\cline { 2 - 5 } & \multicolumn{4}{c}{$\sigma^{2}\left(C_{f}\right)$} \\
I & 0.000039 & 0.000917 & 0.000102 & 0.442224 \\
II & 0.000251 & 0.009366 & 0.000273 & 0.250179 \\
III & 0.000010 & 0.003644 & 0.000166 & 0.749215 \\
IV & 0.000008 & 0.004534 & 0.000076 & 0.686746 \\
V & 0.000019 & 0.000068 & 0.000195 & 0.444950 \\
VI & 0.000023 & 0.000045 & 0.000058 & 0.721707 \\
VII & 0.010898 & 0.032257 & 0.000031 & 0.805210 \\
VIII & 0.009618 & 0.021399 & 0.000009 & 0.352702 \\
\hline
\end{tabular}


Table 2: (Continued).

\begin{tabular}{lcccc}
\hline Experiment & \multicolumn{4}{c}{$\overline{T_{f}}$} \\
\cline { 2 - 5 } I & (i) & (ii) & (iii) & (iv) \\
\cline { 2 - 5 } II & 0.000642 & 0.006438 & 0.474852 & 0.340475 \\
III & 0.000643 & 0.003924 & 0.474737 & 0.389267 \\
IV & 0.000307 & 0.137617 & 0.489661 & 0.355899 \\
V & 0.000310 & 0.102894 & 0.490509 & 0.290770 \\
VI & 0.000237 & 0.335283 & 0.469148 & 0.231000 \\
VII & 0.000237 & 0.366104 & 0.484582 & 0.185903 \\
VIII & 0.002447 & 0.002894 & 0.484342 & 0.042994 \\
& 0.002582 & 0.003393 & 0.484713 & 0.094744 \\
\cline { 2 - 5 } & \multicolumn{4}{c}{$\sigma^{2}\left(T_{f}\right)$} \\
I & $(\mathrm{i})$ & (ii) & $(\mathrm{iii})$ \\
II & 0.000080 & 0.000779 & 0.000010 & 0.005762 \\
III & 0.000079 & 0.000433 & 0.000007 & 0.002972 \\
IV & 0.000031 & 0.000544 & 0.000009 & 0.006885 \\
V & 0.000019 & 0.000585 & 0.000004 & 0.005747 \\
VI & 0.000019 & 0.000005 & 0.000001 & 0.012932 \\
VII & 0.000473 & 0.000532 & 0.000000 & 0.016717 \\
VIII & 0.000497 & 0.000609 & 0.000010 & 0.010095 \\
\hline
\end{tabular}




\section{A Selected spatiotemporal patterns}

In this appendix, we provide illustrations of the spatiotemporal patterns resulting from some of the evolutions based on the update rules of Table 1. In all illustrations, the color white is associated with the 0 state, the color black with the 1 state. All spatiotemporal plots are framed for increased ease of reference.

The first set of illustrations corresponds to some of the evolutions to which Figures 215 refer. They are therefore for infinite cellular automata and correspond to the evolutions of the observed cells, that is, the cells whose states contributed to the entropy calculations. This set is shown in Figures 8 and 9 respectively for the one- and two-dimensional cases.

A similar second set of illustrations depicts the evolution of the same cells, but now under cylindrical boundaries, following our remarks in Section 7 These are given in Figures [10] and [1] respectively for the one- and two-dimensional cases. They are related to the data shown in Table 2 only in principle, because they correspond, for the sake of comparison, to initial configurations that match those used for the infinite cases. 

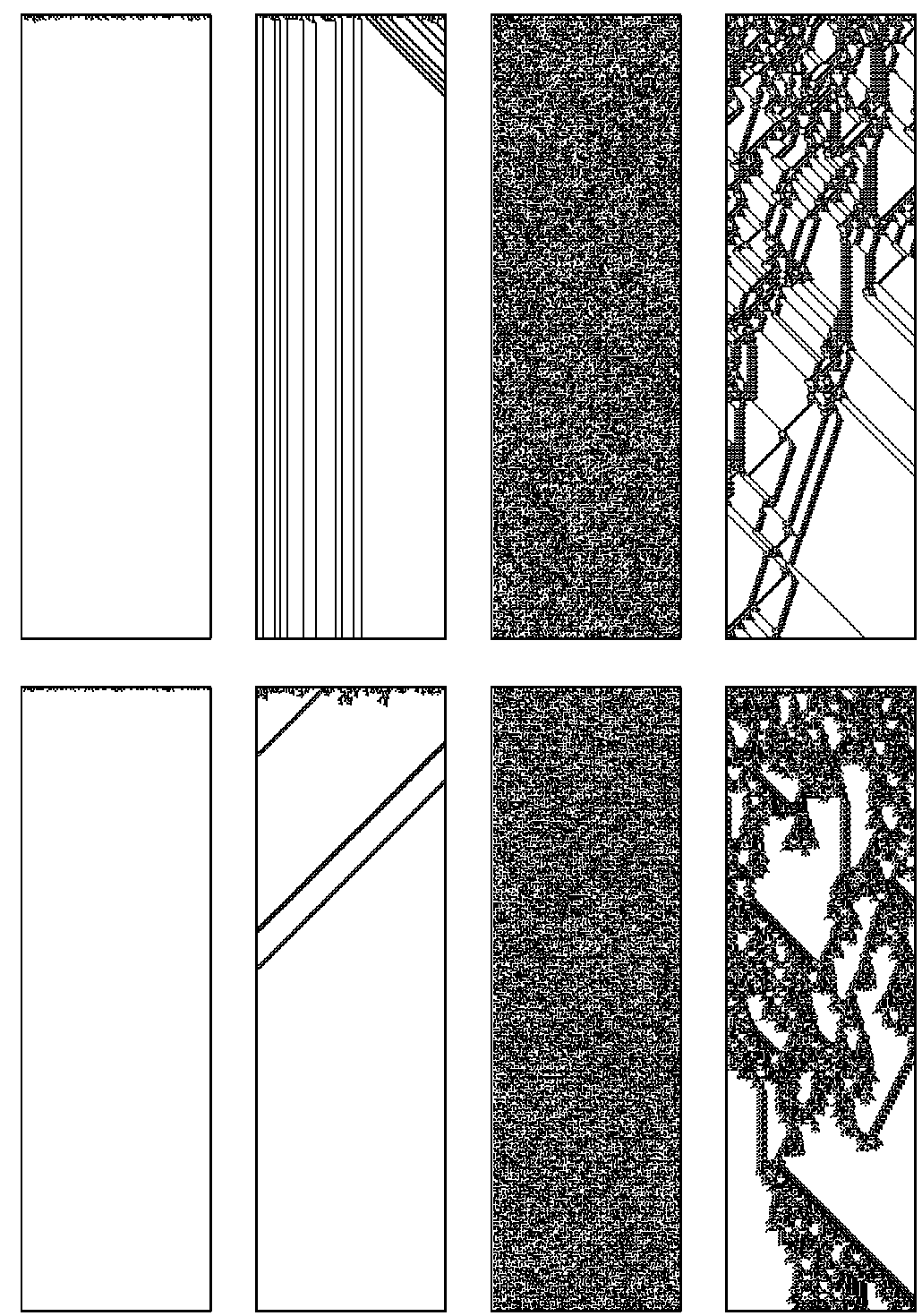

Figure 8: Sample spatiotemporal patterns for the update rules of Table 1 in the infinite, $d=1$ cases with 150 cells observed for 500 time steps. Each plot displays cell states horizontally for each time step; time grows from top to bottom. The topmost row of plots corresponds to $r_{1}=2$, the bottommost to $r_{1}=3$. Within each row, from left to right, the update rules belong each to classes (i)-(iv). 

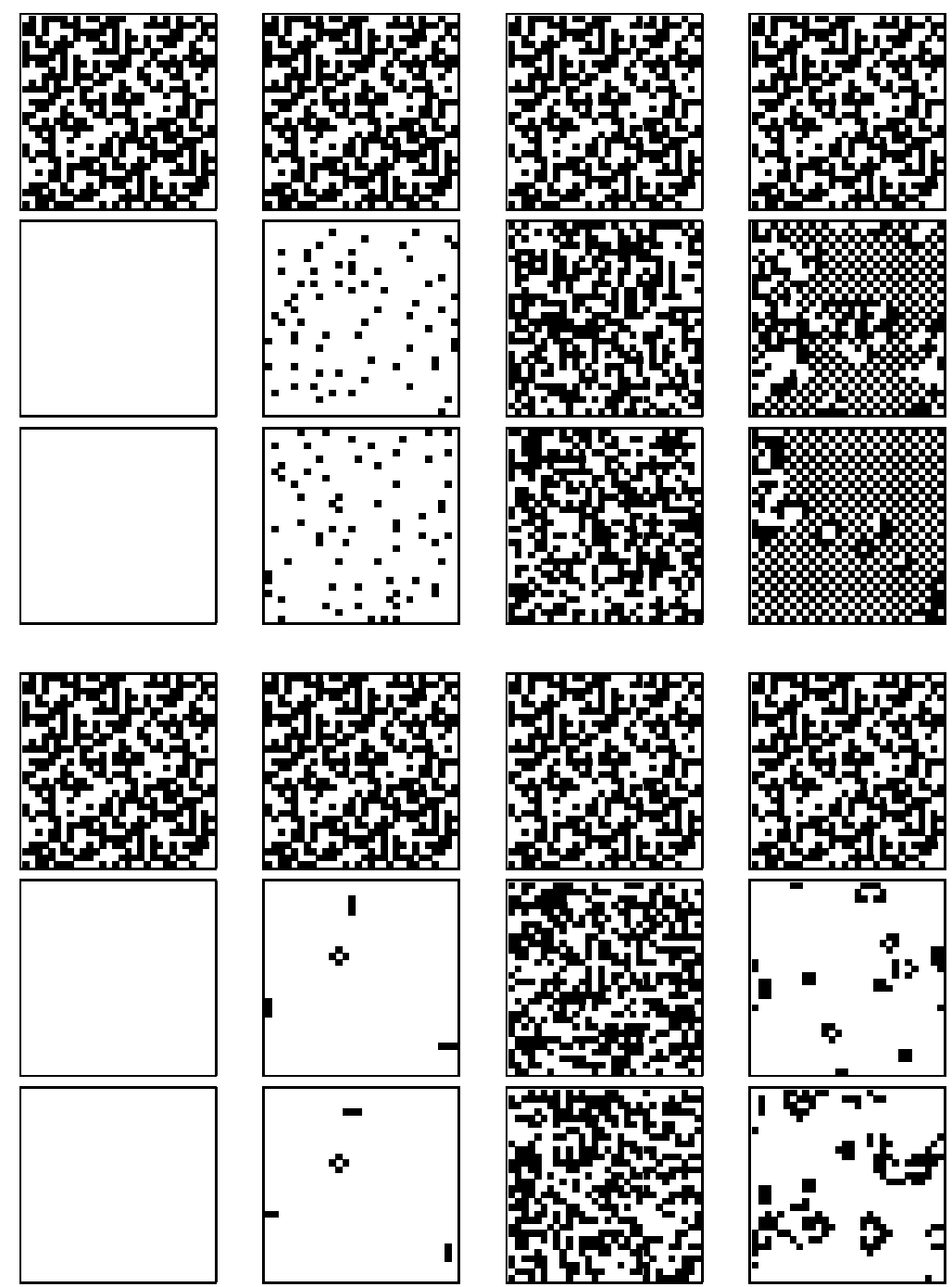

Figure 9: Sample spatiotemporal patterns for the update rules of Table 1 in the infinite, $d=2$ cases with $30 \times 30$ observed cells. Each plot displays a configuration during the evolution of the automaton. The topmost three rows of plots are relative to the von Neumann update rules, the bottommost three rows to the Moore update rules. Within each triple of rows, the topmost row corresponds to $t=0$, the middle one to $t=125$, and the bottommost to $t=250$. Within each row, from left to right, the update rules belong each to classes (i)(iv). 

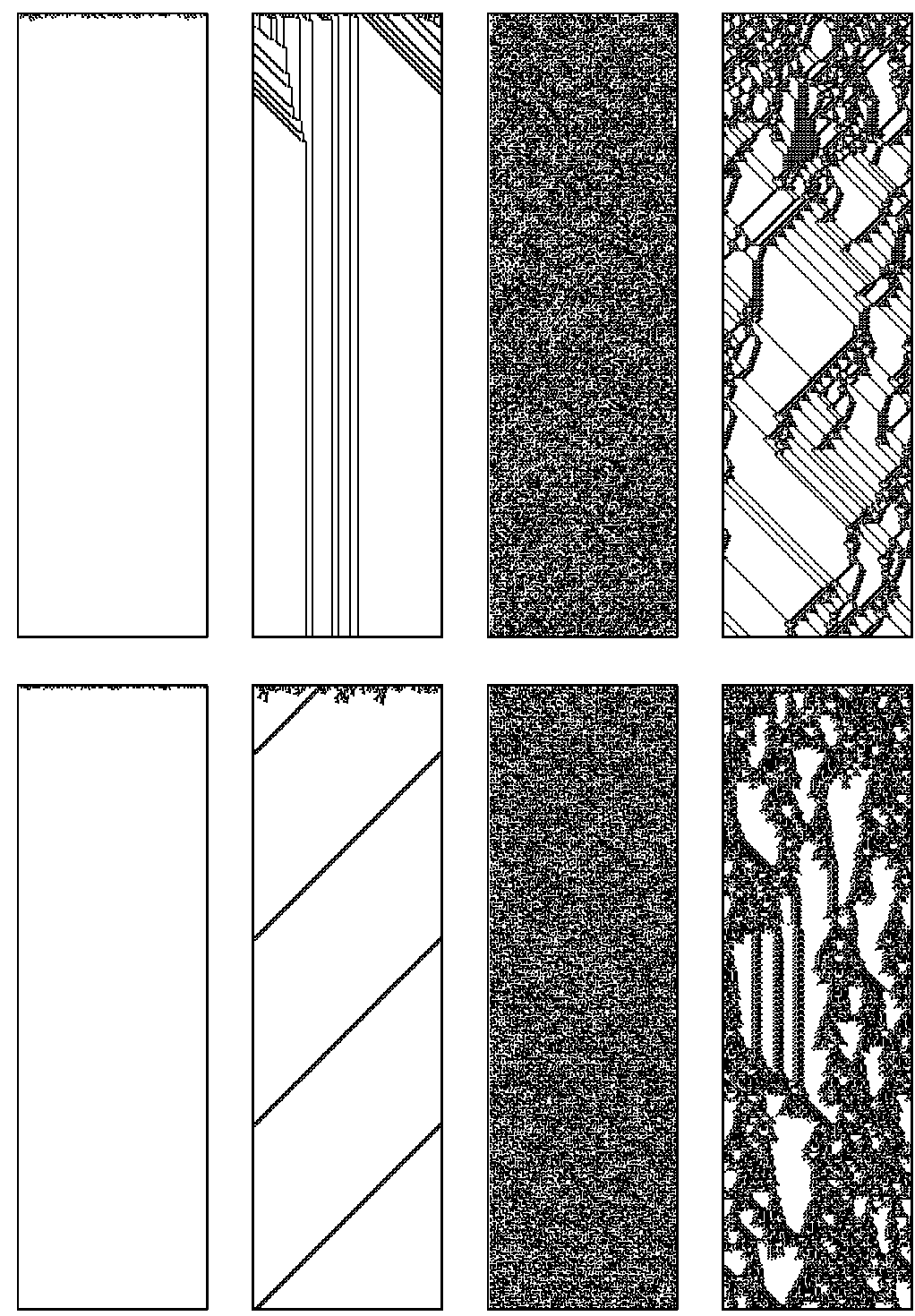

Figure 10: Sample spatiotemporal patterns for the update rules of Table 1 in the cylindrical, $d=1$ cases with 150 cells observed for 500 time steps. Each plot displays cell states horizontally for each time step; time grows from top to bottom. The topmost row of plots corresponds to $r_{1}=2$, the bottommost to $r_{1}=3$. Within each row, from left to right, the update rules belong each to classes (i)-(iv). 

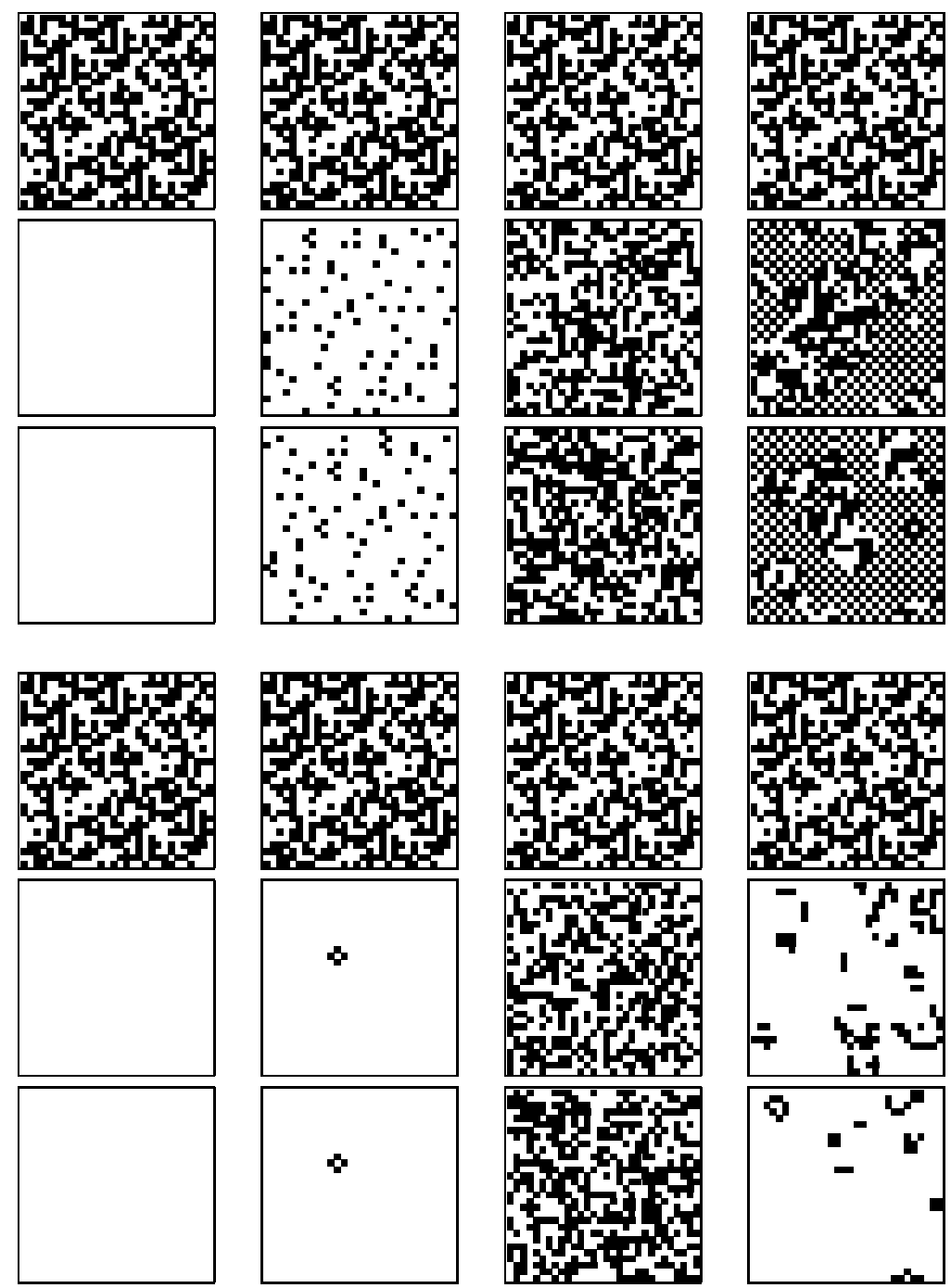

Figure 11: Sample spatiotemporal patterns for the update rules of Table 1 in the cylindrical, $d=2$ cases with $30 \times 30$ observed cells. Each plot displays a configuration during the evolution of the automaton. The topmost three rows of plots are relative to the von Neumann update rules, the bottommost three rows to the Moore update rules. Within each triple of rows, the topmost row corresponds to $t=0$, the middle one to $t=125$, and the bottommost to $t=250$. Within each row, from left to right, the update rules belong each to classes (i)(iv). 


\section{Acknowledgments}

The authors acknowledge partial support from CNPq, CAPES, and a FAPERJ BBP grant.

\section{References}

[1] J. von Neumann, Theory of Self-Reproducing Automata, University of Illinois Press, Urbana, IL, 1966.

[2] M. Kutrib, R. Vollmar, T. Worsch, Introduction to the special issue on cellular automata, Parallel Computing 23 (1997) 1567-1576.

[3] S. Bandini, G. Mauri, R. Serra, Cellular automata: from a theoretical parallel computational model to its application to complex systems, Parallel Computing 27 (2001) 539-553.

[4] S. Wolfram, A New Kind of Science, Wolfram Media, Champaign, IL, 2002.

[5] A. Wuensche, M. Lesser, The Global Dynamics of Cellular Automata, Addison-Wesley, Reading, MA, 1992.

[6] S. Wolfram, Cellular Automata and Complexity, Addison-Wesley, Reading, MA, 1994.

[7] A. Ilachinski, Cellular Automata, World Scientific, Singapore, 2001.

[8] S. Wolfram, Universality and complexity in cellular automata, Physica D 10 (1984) 1-35.

[9] N. H. Packard, S. Wolfram, Two-dimensional cellular automata, Journal of Statistical Physics 38 (1985) 901-946.

[10] J. Kari, Rice's theorem for the limit sets of cellular automata, Theoretical Computer Science 127 (1994) 229-254.

[11] J. Kari, The nilpotency problem of one-dimensional cellular automata, SIAM Journal on Computing 21 (1992) 571-586.

[12] C. G. Langton, Computation at the edge of chaos: phase transitions and emergent computation, Physica D 42 (1990) 12-37.

[13] M. Mitchell, P. T. Hraber, J. P. Crutchfield, Revisiting the edge of chaos: evolving cellular automata to perform computations, Complex Systems 7 (1993) 89-130.

[14] M. Mitchell, J. P. Crutchfield, P. T. Hraber, Dynamics, computation, and the "edge of chaos": a re-examination, in: G. Cowan, D. Pines, D. Meltzer (Eds.), Complexity: Metaphors, Models, and Reality, Addison-Wesley, Reading, MA, 1994, pp. 497-513. 
[15] M. Markus, T. Hahn, I. Kusch, A novel quantification of cellular automata, Parallel Computing 23 (1997) 1635-1642.

[16] G. Braga, G. Cattaneo, P. Flocchini, C. Quaranta Vogliotti, Pattern growth in elementary cellular automata, Theoretical Computer Science 145 (1995) $1-26$.

[17] J.-C. Dubacq, B. Durand, E. Formenti, Kolmogorov complexity and cellular automata classification, Theoretical Computer Science 259 (2001) 271-285.

[18] M. Li, P. Vitányi, An Introduction to Kolmogorov Complexity and Its Applications, 2nd Edition, Springer-Verlag, New York, NY, 1997.

[19] J. E. Hanson, J. P. Crutchfield, Computational mechanics of cellular automata: an example, Physica D 103 (1997) 169-189.

[20] J. E. Savage, Models of Computation, Addison-Wesley, Reading, MA, 1998.

[21] A. Wuensche, Classifying cellular automata automatically: finding gliders, filtering, and relating space-time patterns, attractor basins, and the $Z$ parameter, Complexity 4 (1999) 47-66.

[22] S. Wolfram, Statistical mechanics of cellular automata, Reviews of Modern Physics 55 (1983) 601-644.

[23] M. Wójtowicz, Cellular automata rules lexicon, http://psoup.math. wisc.edu/mcell/ca_rules.html.

[24] M. Gardner, Mathematical games: the fantastic combinations of John Conway's new solitaire game 'Life', Scientific American 223 (1970) 120-123.

[25] E. R. Berlekamp, J. H. Conway, R. K. Guy, Winning Ways for Your Mathematical Plays, Vol. 2, Academic Press, London, UK, 1982.

[26] V. C. Barbosa, An Introduction to Distributed Algorithms, The MIT Press, Cambridge, MA, 1996.

[27] B. Awerbuch, Complexity of network synchronization, Journal of the ACM 32 (1985) 804-823.

[28] V. C. Barbosa, Massively Parallel Models of Computation, Ellis Horwood, Chichester, UK, 1993.

[29] P. Brinch Hansen, Parallel cellular automata: a model program for computational science, Concurrency: Practice and Experience 5 (1993) 425-448.

[30] D. Talia, Cellular processing tools for high-performance simulation, Computer 33 (2000) 44-52.

[31] D. Eppstein, Searching for spaceships, in: R. J. Nowakowski (Ed.), More Games of No Chance, Cambridge University Press, Cambridge, UK, 2002, pp. $433-453$. 
[32] D. Eppstein, Which "Life"-like systems have gliders?, http://www.ics . uci.edu/ eppstein/ca.

[33] Journey into phylogenetic systematics, http://www.ucmp.berkeley.edu/ clad/clad4.html. 